\title{
Temporal evolution of soil moisture statistical fractal and controls by soil texture and regional groundwater flow
}

\author{
Xinye Ji and Chaopeng Shen ${ }^{*}$ \\ Department of Civil and Environmental Engineering \\ The Pennsylvania State University, University Park, PA 16802, USA \\ e-mail: xzj102@psu.edu,cshen@engr.psu.edu; \\ William J. Riley \\ Earth Science Division \\ Lawrence Berkeley National Lab, Berkeley, CA 94720, USA \\ e-mail: wjriley@lbl.gov
}

14 Soil moisture statistical fractal is an important tool for downscaling remotely-sensed observations 15 and has the potential to play a key role in multi-scale hydrologic modeling. The fractal was first 16 introduced two decades ago, but relatively little is known regarding how its scaling exponents 17 evolve in time in response to climatic forcings. Previous studies have neglected the process of 18 moisture re-distribution due to regional groundwater flow. In this study we used a

19 physically-based surface-subsurface processes model and numerical experiments to elucidate the 20 patterns and controls of fractal temporal evolution in two U.S. Midwest basins. Groundwater flow 21 was found to introduce large-scale spatial structure, thereby reducing the scaling exponents $(\tau)$, 22 which has implications for the transferability of calibrated parameters to predict $\tau$. However, the 23 groundwater effects depend on complex interactions with other physical controls such as soil 24 texture and land use. The fractal scaling exponents, while in general showing a seasonal mode that 25 correlates with mean moisture content, display hysteresis after storm events that can be divided into three phases, consistent with literature findings: (a) wetting, (b) re-organizing, and (c) dry-down. Modeling experiments clearly show that the hysteresis is attributed to soil texture, whose "patchiness" is the primary contributing factor. We generalized phenomenological rules for the impacts of rainfall, soil texture, groundwater flow, and land use on $\tau$ evolution. Grid resolution has a mild influence on the results and there is a strong correlation between predictions of $\tau$ from different resolutions. Overall, our results suggest that groundwater flow should be given more consideration in studies of the soil moisture statistical fractal, especially in regions with a shallow water table.

\footnotetext{
* Corresponding author
} 


\section{1. Introduction}

36 Spatial and temporal variations in soil moisture strongly affect many hydrologic processes,

37 including infiltration, evapotranspiration (ET), and subsurface flow [1-8]. Downscaling (inferring

38 fine-scale spatial distribution from coarse-resolution data) soil moisture can help improve the

39 accuracy of modeling these nonlinear, moisture-dependent processes [9-11]. Spatial scaling laws

40 are useful tools for downscaling coarse-grained remotely-sensed observations [12-16].

41 Current techniques for the soil moisture downscaling problem include model-based disaggregation

42 [17], Empirical Orthogonal Function [18], Proper Orthogonal Decomposition Mapping Method

43 [10] and multi-fractal-based approaches [19,20], with which our work is primarily concerned.

44 Since the seminal paper by Rodriguez-Iturbe et al. [1995], it has been widely known that statistical

45 moments of observed soil moisture exhibit power-law decay (e.g., see [11,12,19,22-28]), which

46 resembles a self-similar structure, or fractal, in a statistical sense (for a brief introduction, see

47 Section 2.1). Previous modeling results $[23,29,30]$ also show similar scaling relationships. These

48 scale-invariant relationships and the associated scaling exponents may hold the key to enable

49 estimation of subgrid-scale states from large scale dynamics for modeling purposes [14,31].

50 In addition to providing a tool for observation-based downscaling, the statistical fractal may also

51 contribute to solving the spatial scaling issue in hydrologic modeling. Hydrologic spatial scaling

52 and heterogeneity have for decades been recognized as among the most significant challenges in

53 hydrologic modeling [32]. Scaling problems arise when the models and their associated governing

54 equations are applied at inappropriate spatial scales [33], leading to identifiability (or equifinality)

55 problems [34] for scale-dependent hydrologic models [14,35-38]. Despite the importance of this

56 problem, there is a lack of generalized subgrid scale integration theory. It has been common in

57 other disciplines, e.g., soil structure [39], reactive transport [40,41], modeling of orographic 
58 precipitation [42], geomechanics [43], etc., to employ scale-invariant relationships to infer

59 fine-scale distributions with macro-scale data (e.g., [44-46]). However, we are not aware of the

60 applications of scale-invariance in land surface hydrologic modeling.

61 Despite the potential usefulness of the soil moisture statistical fractal (SMSF), there is limited 62 knowledge of how it evolves in time as watersheds undergo seasonal and daily climatic forcing. 63 Although it is generally understood that a more ,patchy', disconnected, or ,wet islands' pattern has 64 higher spatial ,intermittency' $[20,21]$, leading to higher scaling exponents, no quantitative or 65 qualitative descriptions exist for the continuous temporal evolution of the SMSF, except for its 66 relationships with mean moisture [25,30]. There is even less understanding of the abiotic and 67 biotic controls of the SMSF. Mascaro et al. [2010] used mean moisture and calibrated parameters 68 representing soil texture and vegetation as predictors for the SMSF scaling exponents, but did not 69 examine the impacts of regional groundwater flow. In more humid regions, since groundwater 70 flow likely produces hillslope-scale spatial soil moisture structure [47], we expect groundwater 71 dynamics to exert controls on the SMSF scaling exponents. Whether the mean-moisture-driven 72 formula proposed by Mascaro [2010] works for different geologic and groundwater flow settings 73 is therefore worth investigation. Since an analytical formulation of SMSF scaling exponent 74 evolution considering groundwater flow is not available, empirical evidence can be collected to 75 synthesize patterns and phenomenological laws. Recent discussions encouraged the identification 76 and use of patterns, emergent behaviors, and organizing principles to reduce the dimensionality of 77 the prediction problem [48-53]..

78 The goals of this paper are two-fold: (1) to test the hypothesis that in humid regions, lateral 79 groundwater flow can exert short- and long-term controls on the temporal evolution of the SMSF 80 scaling exponents, comparable to the effects of soil texture, on the $10^{-2} \sim 10^{1} \mathrm{~km}^{2}$ scale. More 
81 specifically, groundwater flow maintains spatial soil moisture structure and thus reduces the 82 values of the SMSF scaling exponents; and (2) to identify and understand how SMSF scaling

83 exponents evolve in response to seasonal and storm-event forcings. The span of spatial scales

84 studied here is chosen because it fills the gap between regional climate modeling and

85 physically-based hydrologic modeling scales, and is therefore of interest to future multi-scale

86 modeling. We analyze the temporal evolution of the SMSF scaling exponents using a tested,

87 physically-based, surface-subsurface processes model applied in two temperate watersheds. We

88 focus on "deep why" explanations of the temporal dynamics and, ultimately, methods for

89 representing heterogeneity in numerical models.

90 In the following, we first provide basic background of the soil moisture statistical multi-fractal.

91 Then we present the impact of groundwater flow on fractal evolution by comparing simulations

92 with and without lateral groundwater flow. Subsequently, we synthesize the temporal evolution

93 patterns of the scaling exponents, explain their responses to seasonal and event forcings, and

94 analyze their controls by soil texture, groundwater flow, and land use. Finally, we show results

95 from different resolutions, as well as a different basin, and discuss the potential for application of 96 the SMSF in large-scale models.

\section{2. Methods}

\section{2.1. Multi-fractal analysis}

99 We denote by $\theta(\lambda)$ the depth-averaged soil moisture (or, more generally, any

100 spatially-distributed variable) in an observation window of size (support scale) $\lambda \times \lambda$ in a

101 domain of size $L \times L$. The $q^{\text {th }}$ order central moment of $\theta(\lambda), S^{q}(\lambda)$, is calculated as

$$
S^{q}(\lambda)=\overline{[\theta(\lambda)-\overline{\theta(\lambda)}]^{q}}
$$


102 where the average operator is taken over the specified domain of size $L \times L$. As discovered from 103 both field observations and numerical simulations, increasing the support scale $\lambda$ at steady state 104 often leads to an approximate power-law decay of $S^{q}$ :

$$
S^{q}(\lambda) \propto \lambda^{-\tau(q)}
$$

105 where $\tau(q)$ are the scaling exponents for each moment. Since the relationship is scale-invariant 106 and self-similar, i.e., $\tau(q) \propto-\frac{\log \left(s^{q}(\lambda)\right)}{\log (\lambda)}$ is a constant for all $\lambda$ (within the $\lambda$ bounds where this 107 relationship holds), the field is fractal in a statistical sense when $0<\tau(q)<2$. At the bounds, $108 \tau(q)=2$ corresponds to a completely independent random field, or white noise, and $\tau(q)=0$ 109 corresponds to a homogeneous field. When $\tau(q) \propto q$, the field is called monofractal and when $110 \tau(q)$ is a nonlinear function of $q$, the field is called multifractal. $\tau(q)$ characterizes the spatial 111 "intermittency" or "irregularities" of the field [25]. On a soil moisture map, a lower 112 "intermittency" field (lower $\tau$ ) presents a more recognizable large-scale pattern with less local 113 concentration. On the other hand, a higher intermittency field, typically associated with drier 114 days, is more random, with less apparent structure and higher values (closer to 2 ) of $\tau$. We use $115 \tau_{z_{1}: z_{2}}(q)$ to denote the $q$-th order scaling exponents calculated for the layer between depths $z_{1}$ 116 and $z_{2}(\mathrm{~cm})$. To improve clarity, we will focus on $\tau_{0: 10}(2)$.

117 For this paper, we run our numerical model (section 2.3) at the finest support scale, $\lambda_{\min }$. 118 Moments at support scale $\lambda$ are obtained by aggregating simulated data from $\lambda_{\min }$.

\section{2.2. Multi-fractal models}

120 Mascaro et al. [25] employed a multi-fractal model (MFM) to describe the theoretical expectation 121 for $\tau$ as a function of $q$, drawn from simulated rainfall fields [54]: 
where $c$ and $\beta$ are fitting parameters. When $\beta=0$, equation (3) describes a monofractal, and

123 a multifractal otherwise. Mascaro et al. [2010] further related $c$ to field mean moisture:

$$
c=c_{\infty}+\delta e^{-\lambda \bar{\theta}}
$$

124 Alternatively, we also attempted the linear model:

$$
c=b+a \bar{\theta}
$$

125 where $c_{\infty}, \delta, \lambda, a$, and $b$ are fitting parameters they attributed to environmental factors such 126 as soils and slopes. Equations (3) and (4) predict that $\tau$ will be higher when the field is drier, 127 consistent with our understanding that drier days are associated with higher intermittency. For 128 each simulated moisture field, a set of $c$ and $\beta$ can be obtained by fitting Equations (3) to 129 extracted $\tau(q)$. Following Mascaro et al. [2010], we fixed $\beta$ while calibrating $c$. Then, we 130 examined the relationships between $\mathrm{c}$ and $\bar{\theta}$ to evaluate whether Equations (4) and (5) are 131 appropriate.

\section{2.3. PAWS+CLM}

133 PAWS (Process-based Adaptive Watershed Simulator) $[47,55,56]$ is a computationally-efficient, 134 physically-based hydrologic model that has been coupled with the community land model, 135 CLM4.0 (hereafter called PAWS+CLM; Figure 1). PAWS+CLM explicitly solves 136 physically-based governing equations for 2D overland flow, 1D flow in channel network, 137 quasi-3D subsurface flow, wetlands, and the dynamic, two-way interactions among these 138 components. The quasi-3D subsurface flow is an approximation to the 3D Richards equation, with 139 vertical soil water flow governed by a series of 1D Richards equation, which are coupled to the 
saturated quasi-3D groundwater flow at the bottom using a physically consistent boundary condition [55]. By reducing the dimensionality of the fully three-dimensional (3D) subsurface problem, the model significantly reduces computational demand with little loss of physics representation. PAWS+CLM emphasizes subgrid mechanisms (e.g., lowland depressions, overland-channel exchange) that attempt to account for lack of spatial resolution.

CLM $[57,58]$ is a comprehensive land surface model encompassing energy and radiation cycles, hydrology, momentum/vapor/heat transfers, soil temperature, freeze and thaw, prognostic vegetation phenology, carbon and nitrogen cycles, and many other processes, through mostly mechanistic descriptions. The energy, momentum, vapor transfers and photosynthesis are solved simultaneously. The soil water flow and groundwater components in CLM have been replaced by PAWS components in the coupled model, while Evapotranspiration (ET) and soil freeze-thaw is calculated by CLM modules and passed to PAWS. Because PAWS is coupled to CLM4.0 (and soon to CLM4.5 [59,60]), we can explicitly investigate interactions between carbon and nitrogen biogeochemical and hydrological dynamics.

PAWS+CLM has shown consistently good performance when compared with many observations from seven basins. The model obtained good Nash-sutcliffe performance coefficient on streamflow (Nash) in US Midwest basins $\left(1140 \mathrm{~km}^{2}\right.$ to $\left.22,260 \mathrm{~km}^{2}\right)$, from 0.6 to 0.75 for calibrated and uncalibrated gages. In the $1837 \mathrm{~km}^{2}$ Clinton River basin [47], we obtained a Nash of 0.61 at the calibration gage and 0.65 at an internal gage, partially influenced by increased missing climatic records after 2006 and the climate station unable to capture a large storm in 2004. Moreover, the $\mathrm{R}^{2}$ between observed and simulated depths to water table (wtd) and soil temperature was 0.66 and 0.97 , respectively. The baseflow was shown in log-scale plots to be better captured than peak flows, yielding higher Nash based on root-square-transformed data. In addition, the soil 
moisture [9], Leaf Area Index (LAI), and ET were shown to be in general agreement with in-situ and satellite-based observations. Similar good performance with different variables were achieved also in the $14,431 \mathrm{~km}^{2}$ Grand River basin and the 22,556 $\mathrm{km}^{2}$ Saginaw River basin [61]. In that study, the model agreed well with terrestrial water storage anomalies from GRACE data [62], and time series of groundwater table $\left(\mathrm{R}^{2}=0.72\right)$, streamflow, ET and soil moisture.

\subsection{Study sites and model setup}

169 In this paper we applied the PAWS+CLM model to two US Michigan basins (Figure 2a-b): the 170 Clinton River Basin $\left(\mathrm{CRB}, 1837 \mathrm{~km}^{2}\right)$ and the Upper Grand River Basin (UG, $\left.4531 \mathrm{~km}^{2}\right)$. The two 171 basins share similar humid continental climate, with strong seasonality and an annual average 172 precipitation input of $\sim 900 \mathrm{~mm} \mathrm{y}^{-1}$, but they have different topography. The UG has very mild 173 terrain (elevation: 195 355 m) and unconsolidated glacial drift surficial geology (moraines and 174 tills) underlain by transmissive Marshall and Saginaw formations (fractured sandstones and shale), 175 while the CRB (175 366 m) has rugged hills in the western half of the basin descending to a flat 176 plain in the eastern half. The CRB also has glacial drift surficial geology, but it is underlain by

177 low-permeability coldwater shale formations. Both CRB and UG have "patchy" soil spatial 178 aggregation pattern (Figure A1 shows the distribution of clay percentages in the CRB), as in many 179 other basins. There are higher percentages of fine-texture lacustrine sediment in the southeast, near 180 the Lake St. Clair, due to lake deposition. As discussed later, we found the soil texture spatial 181 aggregation pattern to be important for the fractal evolution during rainfall hiatus.

182 Throughout the paper, we present results from the CRB, and include discussion about the UG in 183 the Appendix A2. To focus our analysis on the temporal dynamics and basins' SMSF responses, 184 we followed the approach of Gaur and Montanty [63] by using homogenized climatic input from 185 one representative station in each basin (Oxford MI, COOPID 206303 for CRB and East Lansing, 
COOPID 202395 for UG). We acknowledge that spatial patterns in precipitation may have important influence at larger scales on soil moisture distributions [12] and modality [64].

188 We applied PAWS+CLM at $220 \mathrm{~m} \times 220 \mathrm{~m}$ horizontal resolution $\left(\lambda_{\min }\right)$ for the CRB and $400 \mathrm{~m} \times$ $189400 \mathrm{~m}$ resolution for the UG, leading to $256 \times 280$ and $270 \times 240$ grids, respectively. We used 20 190 vertical soil layers with variable thicknesses: layers are thinner near the surface and thicker near 191 the bedrock. Both basins were parameterized using similar input sources. The 30-m resolution 192 National Elevation Dataset (NED) was used to generate cell elevation, river bed elevation, and 193 lowland storage bottom elevation in each gridcell. The 30-m resolution IFMAP 2001 land use and 194 land cover data [65] were aggregated to provide Plant Functional Type (PFT) information, with 195 three dominant land use types (PFTs) in each cell. The 1:24,000 SSURGO dataset [66] was 196 processed by a hierarchical pedotransfer function (PTF), Rosetta [67], to provide water retention 197 and unsaturated vertical conductivity parameters for the vadose zone. We obtained the spatial 198 maps of lateral conductivities of the unconfined aquifer (glacial drift) and depths to bedrock by 199 interpolating well records from the WELLOGIC database [68-70] using Kriging.

200 The model was calibrated using root-transformed Nash-Sutcliffe model performance coefficient 201 by adjusting global multipliers. The procedures and comparisons are given in Shen et al. [47]. A 202 warm-up period was provided from 2001 to 2004, and we only examine the results after 2004.

\section{2.5. Numerical experiments and analysis}

204 We designed a series of numerical experiments highlighting the effects of soil texture and 205 regional groundwater flow on the evolution of the fractal exponents (Table 1). In our previous 206 study in the region, we identified soil texture, groundwater flow, and land use/landcover (LULC) 207 as controls of soil moisture [71]. Therefore, we adopted a factorial experimental design, in which these three factors were enabled or disabled to examine their effects on the SMSF. For LULC, 
we considered both the original input data and a homogenized landscape $(h l)$ with only deciduous broadleaf forest. For soil texture, we considered both the original input and a reshuffled soil texture $(r s)$, in which the positions of the original soil textures were randomly switched in the watershed to eliminate any spatial autocorrelation and correlation with topography and LULC. For each combination of LULC and soil texture inputs, we conducted experiments with (a) groundwater flow enabled $(G W)$; and (b) lateral groundwater flow disabled $(n G W)$ by setting lateral groundwater conductivity, $K$, to 0 . As we modify the $K$ values, the steady state water table position also changes, and it will take many simulation years for the model to re-establish near-equilibrium water table. To reduce the effects of initial data, we considered simulations with the initial water table depths of all simulations set uniformly to the basin-average values $(h d)$.

We developed software utilities to export simulated daily-averaged soil moisture fields and calculate $\tau(q)$ from the fields. Simultaneously calculated were the moments of the fields. For the CRB, we divide the basin into roughly two halves: the western hills and eastern plain. We also distinguish between frozen and non-frozen periods, since the SMSF behaved dramatically different between the two periods (Section 3.1). We define the non-frozen period as the first day with soil ice after Apr $1^{\text {st }}$ to the last non-frozen day before Oct $31^{\text {st }}$. For all the simulations listed in Table 1, we computed scaling exponents and calculated the temporal average and standard deviation for non-frozen period for the entire basin. For the CRB, we also calculate the temporal mean and standard deviation of $\tau(q)$ for the two sub-domains.

229 Several numerical simulations were also designed to demonstrate the temporal evolution of the 230 scaling exponents under idealized situations. Simulation noForcing shows how $\tau$ evolves in the 231 absence of ET and rainfall, thereby allowing the basin to purely re-organize via vertical soil 
moisture movement and groundwater flow. Baseflow and exchange with lowland depressions were still allowed to occur. Such a scenario would obviously never happen, but it helps illustrate 234 the isolated effects of groundwater flow on $\tau_{0: 10}(2)$. In noForcing, following a precipitation event on July 15, 2006, ET or precipitation inputs were stopped from July 16 onwards. To show the typical temporal evolution trajectories of scaling exponents during and after storm events, we ran a hypothetical simulation, storm, which is identical to the control simulation (ctrl) except that precipitation inputs are modified to allow the excursions to evolve without disturbance from new storm events. The precipitation amounts on two starting days have been set to $32 \mathrm{~mm}$ and the storms following these two events were removed to produce two complete, un-interrupted cycles.

241 In addition, we also conducted experiments to examine the impact of grid resolution on our 242 results. The CRB simulations were conducted using $220 \mathrm{~m}$ spatial resolution simulation 243 (resulting in 256x280x20 gridcells). It was currently computationally difficult to run at higher 244 resolutions. Therefore we ran simulation res 2 , which used a coarser grid $\left(\lambda_{\min }=440 \mathrm{~m}\right)$.

\section{3. Results and Discussion}

246 First, we verified the scale-invariance of the simulated soil moisture spatial field by examining 247 the relationships between the spatial moments and the observation window size (Appendix A1). 248 We noted that there were a range of scales where the log-log linear relationship held true, and the 249 upper-limit of the scale-invariant relationship $\left(\lambda_{\max }\right.$, fractal cut-off) was related to the size of the 250 computational domain, so we always calculated the log-log slopes using data below $\lambda_{\max }$. In the 251 following, we first examine how the fractal temporal evolution on a seasonal time scale. Then we 252 demonstrate the influence of groundwater flow on the scaling exponent. Then we focus on how the fractal evolves during rainfall and rainfall hiatus. Subsequently, we show how the scaling 
exponent is related to variance, how grid resolution influences the results, and confirmation results from the UG.

\subsection{Seasonal dynamics of the SMSF scaling exponent}

Time series of the scaling exponents of top $10 \mathrm{~cm}$ soil moisture in the CRB basin have very different dynamics between summer and winter (Figure 3a); similar differences occur in the UG basin (Appendix A2). We identified common seasonal and event-driven patterns in the time series for both basins: (a) a slowly varying seasonal mode during the non-frozen period, in which $\tau_{0: 10}^{2}$ increased from spring to summer, and declined back down during the cooler season when the basin is wetting; (b) short-lived spikes to near maximum values during the winter due to freezing; (c) storm-induced disturbances superimposed on the non-frozen seasonal trend, creating first a small peak, which in a few days reverted to a dip, and then merged into the seasonal mode (Figure 3a subpanel).

Since $\tau_{0: 10}(2)$ is related inversely to the spatial organization of the moisture field, Pattern (a) suggests that the field was most organized (or, "connected") during wetter seasons and that the structure gradually weakened during the summer dry-down, which is consistent with the observations from aircraft-based remotely-sensed data [20]. The non-frozen period fractal exponent is related to seasonally-varying basin states, and in particular basin water storage. This 271 pattern is consistent with the model Mascaro et al. [2010] applied, where mean soil moisture was 272 used a predictor. There was a strong negative correlation between $\tau_{0: 10}(2)$ and non-frozen period 273 basin-average subsurface water storage $(w$, in $\mathrm{cm}$ ) anomaly (defined as water storage minus 274 5-year mean; Figure 2b) in each year except for 2009, with $\mathrm{R}^{2}$ ranging between 0.48 and 0.98. $275 \mathrm{R}^{2}$ in 2009 was only 0.21 , perhaps because this was a particularly wet year, and therefore the 276 effects of dry-down periods were weak. Water storage in this region generally declines during 
Spring and Summer and increases during Fall and Winter [47]. As a result, there are two seasonal limbs to the fractal $\tau_{0: 10}(2)$ dynamics: the "withdrawing limb" and the "storing limb" (marked in Figure 3a). In the withdrawing limb (early Spring to Summer), water is being withdrawn from the basin, and $\tau_{0: 10}(2)$ increases. In the storing limb (end of Summer through Winter), water is being stored in the subsurface and the scaling exponents decrease, with large variations occurring during freezing periods.

The scaling exponent for the entire CRB was consistently less than those for the two sub-domains. This pattern occurred because the entire basin showed more spatial organization than each sub-domain, due to the large contrast in soil moisture between the two sub-domains. Between the two sub-domains, the non-frozen period scaling exponent for the western hills was relatively more static in time and was generally higher than in the plain domain. The eastern plain sub-domain has in general higher soil moisture than the western hills due to basin-scale groundwater flow providing subsidy from the west to the east (Figure A2a-b; also [47]). There is a local spatial pattern within the plain sub-domain: the lower elevation region in the southeast has higher moisture than the northeast. In contrast, the moisture structure in the hills sub-domain is patchier and more isolated, mainly attributable to hillslope-valley contrasts, resulting in higher scaling exponents compared to the plains. The eastern plain domain also showed larger seasonal variation in $\tau_{0: 10}(2)$ due to the shallower groundwater table, which was more strongly influenced by seasonal climate forcings. In a similar finding from field studies, Rosenbaum et al. [72] showed that groundwater-influenced areas (shallow water table) experience a larger range of spatial variance of soil moisture. In a percolation theory framework, Domenico and Laguardia [29] showed that, using another hydrologic model, the percolation probability is influenced by terrain morphology such as bifurcation and elongation ratios. 


\subsection{The impact of groundwater flow on scaling exponents}

\section{3.2.1. Impact on temporal mean and variation of $\tau$}

302 We now examine how the temporally aggregated $\tau$ behavior is influenced by groundwater, 303 LULC, and soil properties. To reduce influence from initial conditions and present a fair 304 comparison between with and without groundwater flow, here we plotted results from the 305 simulations that homogenized the initial depth to water table $(-h d)$. Figure 4 a shows that the 306 temporal mean of non-frozen-period $\tau_{0: 10}(2)$ declined dramatically with increasing physical 307 controls (from right to left). Recall that a $\tau(2)$ value of 2 corresponds to a completely 308 uncorrelated soil moisture distribution (i.e., white noise). For the case with reshuffled soil, 309 homogenized landuse, and disabled groundwater flow, $\tau_{0: 10}(2)=1.97$, therefore representing a 310 nearly random field with no spatial structure. Proceeding in the order of control (cntl), rs-hd, and $311 r s-h l-h d$ (from left to right in Figure 4a), more physical controls were added, and we observed a 312 decreasing trend in $\tau_{0: 10}(2)$. This pattern indicates that spatial structures form in the moisture 313 fields as LULC heterogeneity and spatial soil texture patterns are introduced.

314 For all cases except for the hills sub-domain of the CRB, groundwater flow led to lower scaling 315 exponents. However, the changes caused by groundwater flow were situation-dependent, and not 316 predictable as an additive effect. Based on the control cases, where soil texture patterns were in 317 effect, there was only a difference of $\sim 0.1$ between enabled and disabled groundwater flow. 318 However, based on randomly-distributed-soil cases $(r s-)$, which in general have much higher $319 \tau_{0: 10}(2)$, there were differences in $\tau_{0: 10}(2)$ of $0.19,0.6,0.4$, and 0.22 , respectively, for 320 CRB-hill, CRB-plain, CRB-whole, and UG. This phenomenon may be attributed to how other 321 controlling factors spatially co-vary with groundwater convergence patterns, yielding both 322 correlated and anti-correlated interactions on moisture. Groundwater flow tends to induce higher 
moisture in lowland areas, foothills, and riparian zones, while wetter areas are often found with finer textures (clay) because they are better at retaining moisture. The two factors are sometimes correlated, as there are more clayey soils in the eastern lowland plains, especially near Lake St. Clair (Figure A1). Therefore the soil-texture-dominated pattern is correlated with the groundwater- dominated pattern. However, there can also be conflicting trends. For example, near the stream riparian zones, where groundwater discharges, there is notably less clay. As a result, adding the groundwater flow in the $c t r l$ cases yielded a smaller difference in $\tau$, while adding it to the $r s$ case yielded a much larger difference.

In addition to reducing the mean of $\tau_{0: 10}(2)$, groundwater flow also increased the temporal 332 standard deviation of $\tau_{0: 10}(2)$, while the magnitude of the difference is also case-dependent 333 (Figure 4b). Higher standard deviation indicates stronger temporal variation of soil moisture 334 pattern in response to climate forcings. Especially, for $r s-h l-h d$, when the dominant controls of 335 soil texture and LULC were removed, groundwater alone contributed to the temporal dynamics 336 of $\tau_{0: 10}(2)$. The corresponding temporal standard deviation of $\tau_{0: 10}(2)$ in the $r s-h l-h d-n G W$ 337 cases were all very small, suggesting that the pattern created by groundwater flow is very steady 338 in time, while the soil texture-dominated pattern is very patchy.

\section{3.2.2. Impact on the predictive formula}

340 Since the Mascaro-2010 formulation (Equation 3) does not include groundwater, its effects can

341 be implicitly accounted for during site-specific calibration using the $c \sim \bar{\theta}$ relationship.

342 Therefore, if groundwater flow substantially alters the $c \sim \bar{\theta}$ relationship, we hypothesize that it 343 can affect the transferability of the calibrated parameters. The $c \sim \bar{\theta}$ ( $c$ as in Equation 3) 344 relationships, based on daily data from several simulations, again highlight the complex 
interaction between the groundwater flow effects and other controls (Figure 5). In Figure 5a, the ctrl-hd-nGW points show a simple, generally decreasing relationship between c and $\bar{\theta}$, which can be well described by the linear function in Equation 5. However, enabling groundwater flow (ctrl-hd-GW) introduced many hysteretic excursions which reduced the overall slope of the scattered points. The disturbance effect resulted from groundwater flow patterns not entirely matching the soil texture pattern, as explained in previous section. When soils were reshuffled, however, the $c \sim \bar{\theta}$ completely changed, showing a positive correlation between $c$ and $\bar{\theta}$ in both $r s-h d-n G W$ and $r s-h d-G W$ simulations (Figure 5b). Compared to $r s-h d-n G W, r s-h d-G W$ has increased scattering of $c \sim \bar{\theta}$ points in the lower $c$ range. Further, when the LULC was uniformly set to a deciduous broadleaf forest, the contrast between $G W$ and $n G W$ simulations was more prominent (Figure 5c): $c$ and $\bar{\theta}$ were positively correlated in $r s-h l-h d-G W$ and negatively correlated in $r s-h l-h d-n G W$.

The complete change of behavior from $5 \mathrm{a}$ to $5 \mathrm{~b}$ suggests that soil texture spatial distribution may be the primary factor responsible for the observed negative $c \sim \bar{\theta}$ correlation found in the literature. Consider the rainfall hiatus, when $\bar{\theta}$ decreases (going from the right of panel to the left in any panel in Figure 5). With the original soil texture distribution ( $c t r l$-), the moisture pattern was heavily influenced by small, isolated soil texture patches, leading to higher $c$. On the other hand, in a soil-reshuffled domain, the remaining dominant control besides groundwater flow is the LULC. The smooth, large-scale structure induced by either LULC (e.g., due to canopy shading effects, evapotranspiration) or groundwater flow (due to topography) emerged as drydown occurs, leading to a more organized pattern and thus lower $c$. Consistent with this theory, in Figure 5c, the $c \sim \bar{\theta}$ was almost flat, as no factors introduce patterns, but when 367 groundwater flow was added ( $r s-h l-h d-G W)$, it dominated, leading to positively correlated $c \sim \bar{\theta}$. 


\subsubsection{Impact on the temporal evolution pattern}

In noForcing, the model predicted a slow decline in $\tau$, accompanied by a slow loss of water due

370 to baseflow (Figure 6a). The correlation between mean moisture and $\tau$ is strong after Day 0 . In 371 the absence of mass exchange with the atmosphere, the basin moisture continuously converged

372 to lowland areas (hereafter termed the re-organization phase), leading to a stronger pattern and 373 thus lower $\tau$. In Figure $6 \mathrm{~b}$, the decline in $\tau$ was fastest immediately after D0, and became 374 gradually slower as re-organization proceeded. An analogous experiment with the UG showed a 375 similar pattern (data now shown). Therefore, we can expect the fractal scaling exponent to 376 gradually decrease as the basin releases water during recession periods. However, when the 377 forcing terms (ET and precipitation) are present, the change in $\tau$ depends on the competing 378 effects of inputs and re-organization.

In summary of the results in section 3.2 , soil texture seems to be a dominant controller of the $c \sim \bar{\theta}$ relationship, and, as expected, the soil texture spatial distribution will be a primary contributor to the parameters describing this relationship. However, apart from soil texture and LULC, groundwater flow also introduces noticeable disturbances to the relationship, and characteristically alters the $c \sim \bar{\theta}$ relationship. The calibrated parameters implicitly compensate for the groundwater flow, implying that its transferability may be limited. Therefore, the role of groundwater should be explicitly included in an improved predictive formula.

\subsection{Rainfall-induced hysteresis}

\subsubsection{Typical $\tau$ response to rainfall and rainfall hiatus}

In this section we examine how $\tau$ typically evolves during and after rain events. From Figures

$7 \mathrm{a}$ and $7 \mathrm{~b}$, note that the $\tau_{0: 10}^{2}$ values were not a single-valued function of $\theta_{0: 10}$. Rather, we observed loops and shifts of the $\tau_{0: 10}^{2}$ orbits, indicative of system hysteresis. Hysteresis in the 
coefficient of variation vs. mean moisture state plane was also observed in simulations of an arid system [48,73] and a humid temperature basin [72].

393 These excursion loops can be broadly divided into three phases (Figure 7c): (1) a wetting phase;

394 (2) the subsequent 're-organizing'-dominated phase, shown as the curve bending downward in tau; 395 and (3) a dry-down phase, where $\theta_{0: 10}$ decrease steadily while $\tau_{0: 10}(2)$ increases. After the 396 re-organizing phase finishes, the hysteretic loop merges back onto the seasonal mode. As shown 397 in Figure $7 \mathrm{~b}$ the curve slopes down along an almost constant slope on the $\tau_{0: 10}(2)-\theta_{0: 10}$ plane. 398 A storm event is easily spotted on Figures $7 \mathrm{a}$ and $7 \mathrm{~b}$ as a sudden excursion toward the right 399 (increasing $\theta_{10}$ ), which then gradually bends its way back toward the left, along a separate path, 400 until the next rainfall hits. Figure A2b shows the moisture field on June $28^{\text {th }}$, which was the last 401 day of a dry-down phase. On the next day, June 29th, 2005, 3.56mm of rain fell. The difference 402 in 0-10 $\mathrm{cm}$ soil moisture between these two days $\left(\Delta \theta_{0: 10}\right.$; Figure $\left.8 \mathrm{~b}\right)$ shows higher values in the 403 highland hills in the West and North of the domain. Because these highland hills were much 404 drier after a long drying phase, they were able to absorb more moisture as the storm hit than the 405 lowland plains and local depressions. Therefore, rainfall acted to reduce the variability of the 406 field, thereby very mildly weakening the $\theta_{0: 10}$ spatial structure. Rainfall stopped on July 9th. 407 Over the next three days, $\tau_{0: 10}(2)$ declined and erased the "gain" during the rainfall (Figure 7a). 408 Basin redistribution immediately following rainfall strongly impacts these patterns (Figure 8c). 409 The redistribution is the emergent combination of infiltration, ET, and groundwater lateral flow. 410 However, as we will demonstrate in the next section (3.3.2), the main factor contributing to the 411 hysteresis is the spatial aggregation pattern of soil texture. Ensuing the redistribution, the 412 dry-down phase started and the wet clusters became patchier (Figure 8a shows the change in $413 \theta_{0: 10}$ during a typical dry-down day), resulting in an increasing $\tau_{0: 10}(2)$. 


\subsubsection{Controls of hysteresis and temporal evolution}

415 Orbits from hypothetical simulations clearly established that the soil texture pattern was the main

416 cause of the rainfall-induced hysteresis (Figure 7c-d). The simulations with reshuffled soil

417 texture $(r s)$ did not exhibit any hysteresis. Rather, the orbit showed a positive linear $\tau_{0: 10}(2)$ vs.

$418 \theta_{0: 10}$ relationship, which is contrary to the $c t r l$ case. The $c t r l-n G W$, on the other hand, has a

419 strong hysteresis, and has maintained the negative $\tau_{0: 10}(2)$ vs. $\theta_{0: 10}$ relationship during

420 dry-down. Therefore groundwater could not have been the main cause of hysteresis.

421 We attribute the hysteresis to different soils' infiltration and water retention behavior.

422 Immediately after rainfall, moisture continues to infiltrate and re-organize as driven by pressure 423 gradients. These processes tend to create smooth patterns that lead to lower $\tau$. However, when

424 the re-organization finishes, ET drives the dynamics as the rainfall hiatus continues. Finer texture 425 soils are better at retaining moisture from evaporation and transpiration, and the patchy soil 426 texture aggregation pattern emerges, therefore leading to higher $\tau$. In the $r s$ - simulations, as the 427 patchy aggregation pattern does not exist, $\tau$ decreases during dry-down because of the 428 organizing effects of groundwater flow and LULC.

429 The competing influence of multiple factors on $\tau$ can be qualitatively generalized into several 430 phenomenological laws, as illustrated in Figure 9. During rainfall hiatus, patchy soil texture 431 spatial patterns produces hysteretic loops. Groundwater and LULC always tend to create regional 432 structures and therefore lower $\tau$. These phenomenological laws seem to work for all the cases 433 we simulated for the two basins.

434 In the storm experiments, for the withdrawing limb response (Figure 10a), ET is strong, the 435 re-organization phase is short-lived and under-developed, and the excursion quickly returns to 
the dry-down phase. For the storing limb response (Figure 10b), the re-organization phase is longer, well-developed, the corresponding drop in $\tau$ is significant, and the effect of ET is small. It is through these long re-organization phases that $\tau$ returns to its annual lows. The shapes of the $\tau_{0: 10}(2)$ vs. $\theta_{0: 10}$ curves are qualitatively similar in the withdrawing and storing limbs, with the strengths of ET and precipitation determining the relative differences in the shapes.

Using 8 days of airborne ESTAR soil moisture data and simulation from a land surface model TOPLATS, which was coupled to TOPMODEL, Peters-Lidard et al. [74] also found similar three-phase responses which they termed "atmospheric/infiltration dominated", "transitional" and "drainage/evaporation dominated". During the last phase, they also found the scaling exponent increased. Due to the short length of the campaign, their data was limited. While their model did not explicitly simulate groundwater flow, our results are comparable and our phase-plane clearly illustrated the three phase response to storm as a general phenomenological rule. This fact provided further evidence that the hysteresis was due to soil properties. Moreover, we show that the rainfall response is embedded in the larger-amplitude seasonal cycle. Their study did not explain how physical processes (soil and landuse variability and groundwater flow) each control the evolution of the $\tau$, their interacting effects, and their influence on the predictive formula. Nor did they examine the different behavior of the hysteresis loops in different limbs of the seasonal cycle. The results presented here should improve our understanding of the general rule of fractal evolution.

\subsection{Relationship between mean, variance, and the scaling exponent}

Since an identifiable pattern must be supported by sufficient spatial variability, one may be tempted to think of $\tau$ as a proxy for variance. We show next that this is not the case (Figure 11). Hysteretic loops between $\tau_{0: 10}(2)$ and $\sigma_{10}^{2}$ are noted, suggesting complex and non-unique 
relationships between them. As compared to Figure 7, each storm event in Figure 11 is indicated by a straight movement toward the left with a slight increase in $\tau_{0: 10}(2)$ but a significant drop in $\sigma_{10}^{2}$, in synchrony with increases in the mean moisture (color-coded), which agree with previous studies that showed variance decreasing due to storm events [48]. During the wetting phase, the $\tau_{0: 10}(2)$ and $\sigma_{10}^{2}$ seemed to have a very stable linear relationship, as all wetting events show the same slope. During the re-organization-dominated phases, the orbit returned to the right along a different path than the wetting phases. The correlation between the two variables seemed to break down during the dry-down phases (notwithstanding a few smaller rainfall events), when the variance changed only slightly while $\tau_{0: 10}(2)$ climbs substantially higher. This pattern suggests that $\tau_{0: 10}(2)$ may be better at revealing internal structures of the field than variance. The change in variance seemed to 'stall' during the dry-down process, but the structural pattern continued to evolve. Although a direct mathematical explanation is not presently available, this result highlights the difference between variance and organization.

\subsection{Effect of grid resolution $\left(\lambda_{\min }\right)$}

473 In our resolution refinement analyses, the exponents from higher-resolution simulations (res2)

474 closely followed the patterns from $c t r l$ and the correlation was very strong $\left(\mathrm{R}^{2}=0.975\right)$ (Figure

475 12). However, the fine resolution $\tau$ is always slightly lower than the coarser resolution one. We believe this pattern is due to the ability of the higher-resolution simulation to capture more fine-scale variability in elevation, which leads to more organization of the field (and thus lower $\tau)$. Nevertheless, the temporal pattern discussed in previous sections will still hold had we used the $440 \mathrm{~m}$ simulation results. The strong correlation suggests it is possible to predict fine-resolution $\tau$ using coarser-resolution simulations, after applying some corrections to 481 remove the negative bias, making a fractal-based multi-scale modeling approach more tractable. 


\subsection{Implications for potential predictive formula for $\tau$}

Previous observational studies reported higher scaling exponent on drier days, and proposed that scaling exponents can be related to mean moisture and ancillary environmental predictors [25]. Indeed, this is a promising direction and our results also suggest that fractal exponents may be controlled by environmental factors. However, we note that caution must be exercised when prescribing a relationship between $\tau$ and mean moisture. As the hysteretic loops and seasonal limbs in Figures 7 suggest, not only is there no simple 1-to-1 mapping between $\tau$ and $\theta$, their relationship may also depend on seasonal basin water storage and phase position in the excursion. A static regression equation is therefore not likely to accurately capture these dynamics.

\section{Limitations and future work}

This study did not examine the relative impact of rainfall, humidity, wind speed, solar radiation, and temperature spatial variability on basin fractal evolution. It is known that rainfall fields also show multifractal scaling [54], making interpretation of the impacts of watershed surface and subsurface properties on fractal scaling exponents substantially more complex. However, we argue that understanding the fractal exponent responses related to land properties is a first step toward understanding the overall system responses. Climate variability can play a significant role on the meso-scale $(10 \mathrm{~km})$, but there is still substantial sub-cell heterogeneity that can be addressed by fractal-based multi-scale approaches.

As in other modeling studies, the results of this study are influenced by the model employed.

Although we have utilized a well-tested physically-based hydrologic land-surface model,

PAWS+CLM, all models have simplifications that may impact their predictions. For example, PAWS+CLM makes a simplifying assumption, for computational reasons, that moisture does not flow laterally above the fluctuating water table. This simplification may introduce errors where a 
shallow perched water table is important. Our current model also does not simulate

hydrophobicity or soil shrinkage and swelling, which could be important for soil water bypass

flow, and influence upslope soil water recharge. We expect different hydrologic models will

produce somewhat different responses in fractal evolution, but do not expect qualitative changes.

Admittedly, the patterns we discovered through numerical studies are far from being applicable

in a predictive formula, but it is an important step toward characterizing soil moisture fractal

evolution. Synthesis of observed patterns and behaviors (and, in our case, patterns from

numerical simulations) is an important approach toward a coherent body of knowledge [75]. For

example, given that the scaling exponents better reflect the moisture organization than variance

514 (section 3.4), future work may consider using this knowledge and the observed scaling exponents

515 (e.g., from satellite observations) to infer the phase of soil moisture dynamics.

\section{5. Conclusions}

517 In general, groundwater flow introduces large-scale spatial patterns in near-surface soil moisture 518 and therefore reduces the fractal scaling exponents and increases their temporal variation.

519 However, the complexity lies with the interaction and covariation between groundwater and other 520 physical controls, which may limit the transferability of calibrated parameters. These interactions 521 make it difficult to propose a general predictor-based formula for the scaling exponents.

We identified a seasonal mode that is related to seasonal variation in basin water storage, while storm events induce hysteretic excursion loops which can be divided into wetting,

524 re-organization-dominated, and dry-down-dominated phases. A predictive formula for the scaling 525 exponents will likely need to include phase-dependent terms. The responses of $\tau$ to climatic 526 forcing seem to generally follow a set of phenomenological rules. All of our simulations in the two basins agree with these rules, and their applicability can be examined in other basins. 
Although in this study we made no attempt to propose governing equations to describe these rules, they have the potential to help us develop such a predictive formula. The hysteresis is shown to be due to heterogeneous infiltration, basin self-organization of moisture, and, more importantly, soil texture spatial aggregation. The more "patchy" the soil texture, the higher the fractal scaling exponents will be. Topography and groundwater flow play important roles in modulating the fractal evolution.

\section{Appendices}

\section{A1. Evidence of scale invariance in simulated soil moisture fields}

The clay percentages in the CRB are been shown in Figure A1. As we can see, the spatial aggregation of soil texture tends to be patchy, showing many small clumps. This is an important factor determining fractal evolution. To illustrate the validity of the scale-invariance assumption, we show the spatial fields of soil moisture (liquid phase only) on two days of the simulation in the CRB and the related power-law decay of spatial moments in Figure A2, for both the whole basin and the sub-domains. Although both panels show a large-scale moisture gradient, the contrast is larger in Figure A2b (Dec 01, 2005). The eastern lowland on average was much wetter than the

543 Western hills due to groundwater lateral redistribution. The soil moisture field on the lowland was 544 also smoother. June 28, 2005 represents a day with large $\tau_{0: 10}(2)$, when soil moisture was near the 545 driest in the year. Dec 02, 2005 represents a day with low $\tau_{0: 10}(2)$, showing a stronger large-scale 546 contrast between the western half and eastern halves of the basin.

547 In the whole basin and the two sub-domains there are a range of scales where the log-log linear 548 relationship holds true. We also notice an upper-limit of the scale-invariant relationship $\left(\lambda_{\max }\right.$, 549 fractal cut-off), indicated by the dark dashed lines, beyond which the scaling curves deviate from a linear relationship. For the CRB (basin area of $1837 \mathrm{~km}^{2}$ ), $\lambda_{\max }$ has been determined to be 
around $3520 \mathrm{~m}$ after examining the scale-invariance plot (Figure A2) from a random sample of 30 days of simulation. In addition, for the whole time series, we have verified there is no statistically significant departure from the linear trend within the fractal cut-off. On each day, a robust regression with the Huber weighting function [76] was applied to the data. The Robust regression iteratively reweights the data points to check for violation of assumptions of least squares fit, e.g., outliers, or nonlinearity, which will be assigned lower weights in the fitting. Thus, a low weight (here we take as <0.9) for a data point indicates that it fell off the scale-invariance relationship.

From the time series of the weights (Figure A2e), the $\lambda=7080 \mathrm{~m}$ point was always considered an outlier (due to nonlinearity); the $\lambda=5280 \mathrm{~m}$ point was assigned low weight only during driest parts of the year, and the weight for point $\lambda=3520$ was always above 0.98 . Therefore, it was reasonable to set $\lambda_{\max }$ as $3520 \mathrm{~m}$. Similarly, for the UG ( $4531 \mathrm{~km}^{2}$, data not shown here), $\lambda_{\max }$ is around $9000 \mathrm{~m}$. We believe this divergence from linear scaling is due to the limited size of our basins, and not an intrinsic property of the scaling structure. All scaling exponents are estimated from scales $\in\left[\lambda_{\min }, \lambda_{\max }\right]$.

\section{A2. Results from the Upper Grand}

566 The UG basin has similar climatic forcing but different topography than the CRB. Figure A3a 567 shows the time series of $\tau_{0: 10}(2)$ from UG and Figure A3b shows the $\tau_{0: 10}(2) \sim \theta_{0: 10}$ plot in 5682005 , also from a weather-homogenized simulation. Generally, the results were similar to those

569 from the CRB, with some differences. First, $\tau_{0: 10}(2)$ was much larger than the CRB, but with a 570 tighter range, varying between 0.95 and 1.05. We attribute the larger $\tau$ values and smaller 571 variation to (a) much patchier soil texture, especially in the upper basin; and (b) lack of large-scale 572 topography patterns in the UG, which does not have a hill-plain contrast as the CRB does. As a result, the large-scale pattern was weak and moisture patterns in the UG were more spatially 
intermittent and appear more like scattered islands [21] compared to the CRB. The model did predict seasonal withdrawing and storing limbs, and the significant impacts by freezing. The model also predicted rainfall-induced excursions. Figure A3b shows many rainfall-induced excursions between May $30^{\text {th }}$ and Oct $18^{\text {th }}, 2005$, which are similar to those observed in the CRB. The rises accompanying the storm events were larger than those in the CRB, perhaps due to the milder terrain, which can lead to stronger ,homogenizing' effects by storms.

580 In the UG, freezing also caused more obvious downward spikes when only a clustered fraction of 581 the domain froze, and created a temporary spatial pattern. For example, our model predicted that 582 soil covered by forest froze earlier than urban and agricultural areas. When only the forested 583 areas froze in the UG simulations, the soil moisture pattern created a downward spike in $584 \tau_{0: 10}(2)$

586 Acknowledgements. This research was supported by Office of Biological and Environmental 587 Research of the US Department of Energy to Pennsylvania State University under Contract No. 588 DE-SC0010620; WJR was also supported by US Department of Energy to Lawrence Berkeley 589 National Laboratory under Contract No. DE-AC02-05CH11231. Cartographical assistance from 590 Kurt M. Smithgall is appreciated. 
593 Table 1. Codes of simulations.

\begin{tabular}{|c|c|c|}
\hline Abbreviations & Procedures & Objectives \\
\hline ctrl & $\begin{array}{l}\text { Use the baseline parameters; weather input in } \\
\text { the basin was set as from one single station }\end{array}$ & $\begin{array}{l}\text { To show seasonal and rainfall-induced } \\
\text { dynamics with less noise }\end{array}$ \\
\hline$r s$ & $\begin{array}{l}\text { The soil textures were reshuffled and } \\
\text { randomly placed in the domain, breaking } \\
\text { existing spatial autocorrelation }\end{array}$ & $\begin{array}{l}\text { To demonstrate the effects of soil texture } \\
\text { pattern on scaling exponents }\end{array}$ \\
\hline$h l$ & $\begin{array}{l}\text { LULC was uniformly set to Deciduous } \\
\text { Broadleaf Forest (DBF) }\end{array}$ & $\begin{array}{l}\text { To show the effects of LULC spatial pattern } \\
\text { on scaling exponents }\end{array}$ \\
\hline hd & $\begin{array}{l}\text { Initial water table was homogenized to the } \\
\text { average water table depths in the basin }\end{array}$ & $\begin{array}{l}\text { To reduce the impact of initial conditions on } \\
-G W \text { and }-n G W \text { comparisons }\end{array}$ \\
\hline storm & $\begin{array}{l}\text { Based on } c t r l \text {, some precipitation events were } \\
\text { removed }\end{array}$ & $\begin{array}{l}\text { To present two prototypical rainfall-induced } \\
\text { cycles }\end{array}$ \\
\hline noForcing & $\begin{array}{l}\text { Based on ctrl, source terms (ET and } \\
\text { precipitation) were removed. }\end{array}$ & To show effect of basin self-organization \\
\hline res2 & Based on $c t r l$, grid resolution reduced in half & $\begin{array}{l}\text { To show the effect of grid resolution on } \\
\text { analyzed scaling exponents }\end{array}$ \\
\hline$n G W$ & $\begin{array}{l}\text { Lateral groundwater flow was disabled by } \\
\text { setting aquifer conductivity, } \mathrm{K} \text {, to } 1 \mathrm{e}-14 \mathrm{~m} \\
\text { day }^{-1}\end{array}$ & $\begin{array}{l}\text { To show the effect of having no lateral } \\
\text { groundwater flow }\end{array}$ \\
\hline$G W$ & $\begin{array}{l}\text { Groundwater flow was enabled and } \mathrm{K} \text { was } \\
\text { given the original input values }\end{array}$ & \\
\hline
\end{tabular}




\section{References}

[1] Paschalis A, Fatichi S, Molnar P, Rimkus S, Burlando P. On the effects of small scale space-time variability of rainfall on basin flood response. J Hydrol 2014.

[2] Heathman GC, Cosh MH, Merwade V, Han E. Multi-scale temporal stability analysis of surface and subsurface soil moisture within the Upper Cedar Creek Watershed, Indiana. CATENA 2012;95:91-103.

[3] Brocca L, Tullo T, Melone F, Moramarco T, Morbidelli R. Catchment scale soil moisture spatial-temporal variability. J Hydrol 2012;422-423:63-75.

[4] Wilson DJ. Identifying and quantifying sources of variability in temporal and spatial soil moisture observations. Water Resour Res 2004;40:1-11.

[5] Entin JK, Robock A, Vinnikov KY, Hollinger SE, Liu S, Namkhai A. Temporal and spatial scales of observed soil moisture variations in extratropics. J Geophys Res 2000;105:11865-77.

[6] Seneviratne SI, Corti T, Davin EL, Hirschi M, Jaeger EB, Lehner I, et al. Investigating soil moisture-climate interactions in a changing climate: A review. Earth-Science Rev 2010;99:125-61.

[7] Western AW, Grayson RB, Blöschl G. Scaling of soil moisture: A hydrologic perspective. Annu Rev Earth Planet Sci 2002;30:149-80.

[8] Famiglietti JS, Ryu D, Berg AA, Rodell M, Jackson TJ. Field observations of soil moisture variability across scales. Water Resour Res 2008;44.

[9] Riley WJ, Shen C. Characterizing coarse-resolution watershed soil moisture heterogeneity using fine-scale simulations. Hydrol Earth Syst Sci 2014;18:2463-83.

[10] Pau GSH, Bisht G, Riley WJ. A reduced-order modeling approach to represent subgrid-scale hydrological dynamics for land-surface simulations: application in a polygonal tundra landscape. Geosci Model Dev 2014;7:2091-105.

[11] Wood EF. Scale analyses for land-surface hydrology. In: Sposito G, editor. Scale Depend. scale invariance Hydrol., Cambridge University Press; 1998, p. 1-29.

[12] Ryu D, Famiglietti JS. Multi-scale spatial correlation and scaling behavior of surface soil moisture. Geophys Res Lett 2006;33:L08404.

[13] Choi M, Jacobs JM. Spatial soil moisture scaling structure during Soil Moisture Experiment 2005. Hydrol Process 2011;25:926-32. 
[14] Gebremichael M, Rigon R, Bertoldi G, Over TM. On the scaling characteristics of observed and simulated spatial soil moisture fields. Nonlinear Process Geophys 2009; 16:141-50.

[15] Western AW, Blöschl G. On the spatial scaling of soil moisture. J Hydrol 1999;217:203-24.

[16] Shin Y, Mohanty BP. Development of a deterministic downscaling algorithm for remote sensing soil moisture footprint using soil and vegetation classifications. Water Resour Res 2013;49:6208-28.

[17] Ines AVM, Mohanty BP, Shin Y. An unmixing algorithm for remotely sensed soil moisture. Water Resour Res 2013;49:408-25.

[18] Busch FA, Niemann JD, Coleman M. Evaluation of an empirical orthogonal function-based method to downscale soil moisture patterns based on topographical attributes. Hydrol Process 2012;26:2696-709.

[19] Kim G, Barros AP. Downscaling of remotely sensed soil moisture with a modified fractal interpolation method using contraction mapping and ancillary data. Remote Sens Environ 2002;83:400-13.

[20] Mascaro G, Vivoni ER, Deidda R. Downscaling soil moisture in the southern Great Plains through a calibrated multifractal model for land surface modeling applications. Water Resour Res 2010;46:1-18.

[21] Rodriguez-Iturbe I, Vogel GK, Rigon R, Entekhabi D, Castelli F, Rinaldo A. On the spatial organization of soil moisture fields. Geophys Res Lett 1995;22:2757-60.

[22] Biswas A, Zeleke TB, Si BC. Multifractal detrended fluctuation analysis in examining scaling properties of the spatial patterns of soil water storage. Nonlinear Process Geophys 2012;19:227-38.

[23] Laguardia G, Di Domenico A, Gebremichael M. On the Critical Behaviour of Observed and Simulated Spatial Soil Moisture Fields during SGP97. Remote Sens 2010;2:2097-110.

[24] Di Domenico A, Laguardia G, Fiorentino M. Capturing critical behaviour in soil moisture spatio-temporal dynamics. Adv Water Resour 2007;30:543-54.

[25] Mascaro G, Vivoni ER, Deidda R. Soil moisture downscaling across climate regions and its emergent properties. J Geophys Res 2011;116:1-19.

[26] Syed TH, Famiglietti JS, Rodell M, Chen J, Wilson CR. Analysis of terrestrial water storage changes from GRACE and GLDAS. Water Resour Res 2008;44:W02433. 
[27] Hu Z, Islam S, Cheng Y. Statistical characterization of remotely sensed soil moisture images. Remote Sens Environ 1997;61:310-8.

665

666

667

668

669

[28] Pelletier JD, Malamud BD, Blodgett T, Turcotte DL. Scale-invariance of soil moisture variability and its implications for the frequency-size distribution of landslides. Eng Geol 1997;48:255-68.

[29] Di Domenico A, Laguardia G. On the relations between critical behaviour of soil moisture spatio-temporal dynamics and catchment descriptors. CATENA 2011;86:49-56.

[30] Manfreda S, McCabe MF, Fiorentino M, Rodríguez-Iturbe I, Wood EF. Scaling characteristics of spatial patterns of soil moisture from distributed modelling. Adv Water Resour 2007;30:2145-50.

[31] Kumar P. A multiple scale state-space model for characterizing subgrid scale variability of near-surface soil moisture. Geosci Remote Sensing, IEEE Trans 1999;37.

[32] Beven K. Linking parameters across scales: Subgrid parameterizations and scale dependent hydrological models. Hydrol Process 1995;9:507-25.

[33] Bloschl G, Sivapalan M. Scale issues in hydrological modelling: A review. Hydrol Process 1995;9:251-90.

[34] Beven K. A manifesto for the equifinality thesis. J Hydrol 2006;320:18-36.

[35] Arrigo JAS, Salvucci GD. Investigation hydrologic scaling: Observed effects of heterogeneity and nonlocal processes across hillslope, watershed, and regional scales. Water Resour Res 2005;41:W11417.

[36] Nykanen DK, Foufoula-Georgiou E. Soil moisture variability and scale-dependency of nonlinear parameterizations in coupled land- atmosphere models. Adv Water Resour $2001 ; 24$.

[37] Wood EF. Effects of soil moisture aggregation on surface evaporative fluxes. J Hydrol 1997;190:397-412.

[38] Vivoni ER, Entekhabi D, Bras RL, Ivanov VY. Controls on runoff generation and scale-dependence in a distributed hydrologic model. Hydrol Earth Syst Sci 2007;11:1683-701.

[39] Perrier EMA, Bird NRA. The PSF model of soil structure: a multiscale approach. In: Pachepsky Y, Radcliffe DE, Selim HM, editors. Scaling Methods Soil Phys., 2003.

[40] Shen C, Phanikumar MS. An efficient space-fractional dispersion approximation for stream solute transport modeling. Adv Water Resour 2009;32:1482-94. 
[41] Benson D a., Meerschaert MM, Revielle J. Fractional calculus in hydrologic modeling: A numerical perspective. Adv Water Resour 2012.

697

698

699

700

701

702

703

704

[42] Nogueira M, Barros AP, Miranda PMA. Multifractal properties of embedded convective structures in orographic precipitation: toward subgrid-scale predictability. Nonlinear Process Geophys 2013;20:605-20.

[43] Arcady V. Dyskin. Continuum Fractal Mechanics of The Earth's Crust. In: Donnellan A, Mora P, Matsu'ura M, Yin X, editors. Comput. Earthq. Sci. Part I, Basel: Birkhäuser Basel; 2004.

[44] Chen Q, Gunzburger M, Ringler T. A Scale-Invariant Formulation of the Anticipated Potential Vorticity Method. Mon Weather Rev 2011;139:2614-29.

[45] E W, Yue XY. Heterogeneous Multiscale Method for Locally Self- Similar Problems. Comm Math Sci 2004;2:137-44.

[46] Chorin AJ. Averaging and renormalization for the Korteveg-deVries-Burgers equation. Proc Natl Acad Sci U S A 2003;100:9674-9.

[47] Shen C, Niu J, Phanikumar MS. Evaluating controls on coupled hydrologic and vegetation dynamics in a humid continental climate watershed using a subsurface - land surface processes model. Water Resour Res 2013;49:2552-72.

[48] Ivanov VY, Fatichi S, Jenerette GD, Espeleta JF, Troch P a., Huxman TE. Hysteresis of soil moisture spatial heterogeneity and the "homogenizing" effect of vegetation. Water Resour Res 2010;46:1-15.

[49] Schaefli B, Harman CJ, Sivapalan M, Schymanski SJ. HESS Opinions: Hydrologic predictions in a changing environment: behavioral modeling. Hydrol Earth Syst Sci 2011;15:635-46.

[50] Sivapalan M, Thompson SE, Harman CJ, Basu NB, Kumar P. Water cycle dynamics in a changing environment: Improving predictability through synthesis. Water Resour Res 2011;47:1-7.

[51] Thompson SE, Harman CJ, Troch PA, Brooks PD, Sivapalan M. Spatial scale dependence of ecohydrologically mediated water balance partitioning: A synthesis framework for catchment ecohydrology. Water Resour Res 2011;47:W00J03.

[52] Vivoni ER. Spatial patterns, processes and predictions in ecohydrology: integrating technologies to meet the challenge. Ecohydrology 2012;5:235-41.

[53] Wagener T, Sivapalan M, Troch P a., McGlynn BL, Harman CJ, Gupta H V., et al. The future of hydrology: An evolving science for a changing world. Water Resour Res 2010;46:1-10. 
[54] Deidda R. Multifractal analysis and simulation of rainfall fields in space. Phys Chem Earth, Part B Hydrol Ocean Atmos 1999;24:73-8.

Shen C, Phanikumar MS. A process-based, distributed hydrologic model based on a large-scale method for surface-subsurface coupling. Adv Water Resour 2010;33:1524-41.

[56] Shen C, Niu J, Fang K. Quantifying the effects of data integration algorithms on the outcomes of a subsurface-land surface processes model. Environ Model Softw 2014;59:146-61.

[57] Lawrence DM, Oleson KW, Flanner MG, Thornton PE, Swenson SC, Lawrence PJ, et al. Parameterization improvements and functional and structural advances in Version 4 of the Community Land Model 2011.

[58] Oleson K, Lawrence DM, Bonan GB, Flanner M, Kluzek E, Lawrence P, et al. Technical description of version 4.0 of the Community Land Model (CLM). NCAR Technical Note, NCAR/TN-478+STR.; 2010.

[59] Koven CD, Riley WJ, Subin ZM, Tang JY, Torn MS, Collins WD, et al. The effect of vertically resolved soil biogeochemistry and alternate soil $\mathrm{C}$ and $\mathrm{N}$ models on $\mathrm{C}$ dynamics of CLM4. Biogeosciences 2013;10:7109-31.

[60] Tang JY, Riley WJ, Koven CD, Subin ZM. CLM4-BeTR, a generic biogeochemical transport and reaction module for CLM4: model development, evaluation, and application. Geosci Model Dev 2013;6:127-40.

[61] Niu J, Shen C, Li S-G, Phanikumar MS. Quantifying storage changes in regional Great Lakes watersheds using a coupled subsurface-land surface process model and GRACE, MODIS products. Water Resour Res 2014;50:7359-77.

[62] JPL. GRACE monthly mass grids - land. Jet Propuls Lab GRACE Tellus, Data Process by Sean Swenson 2014.

[63] Gaur N, Mohanty BP. Evolution of physical controls for soil moisture in humid and subhumid watersheds. Water Resour Res 2013;49:1244-58.

[64] Ryu D, Famiglietti JS. Characterization of footprint-scale surface soil moisture variability using Gaussian and beta distribution functions during the Southern Great Plains 1997 (SGP97) hydrology experiment. Water Resour Res 2005;41.

[65] MDNR. 2001 IFMAP/GAP Lower Peninsula Land Cover. Michigan Dep Nat Resour 2010.

[66] NRCS. SSURGO Soil Survey Geographic Database. Nat Resour Conserv Serv 2010. 
[67] Schaap MG, Leij FJ, van Genuchten MT. Rosetta: a Computer Program for Estimating Soil Hydraulic Parameters With Hierarchical Pedotransfer Functions. J Hydrol 2001;251:163-76.

[68] GWIM. State of Michigan Public Act 148 Groundwater Inventory and Mapping Project (GWIM). Technical report 2010.

[69] Oztan M, Li SG. GIS-enabled modeling of Michigan's groundwater systems. Michigan State University, 2011.

[70] Simard A, Li S. Predicting groundwater flow and transport using Michigan's statewide wellogic database. Michigan State University, 2007.

[71] Shen C, Niu J, Phanikumar M. Evaluating controls on coupled hydrologic and vegetation dynamics in a humid continental climate watershed using a subsurface-land surface processes model. Water Resour Res 2013.

[72] Rosenbaum U, Bogena HR, Herbst M, Huisman JA, Peterson TJ, Weuthen A, et al. Seasonal and event dynamics of spatial soil moisture patterns at the small catchment scale. Water Resour Res 2012;48.

[73] Vivoni ER, Rodríguez JC, Watts CJ. On the spatiotemporal variability of soil moisture and evapotranspiration in a mountainous basin within the North American monsoon region. Water Resour Res 2010;46.

[74] Peters-Lidard C., Pan F, Wood E. A re-examination of modeled and measured soil moisture spatial variability and its implications for land surface modeling. Adv Water Resour 2001;24:1069-83.

[75] Thompson SE, Harman CJ, Schumer R, Wilson JS, Basu NB, Brooks PD, et al. Patterns, puzzles and people: implementing hydrologic synthesis. Hydrol Process 2011;25:3256-66.

785 [76] Huber PJ. Robust Statistics. Hoboken, NJ, USA: John Wiley \& Sons, Inc.; 1981. 




Figure 1. Definition sketch of PAWS+CLM. This Figure is modified from Figure 1 in [55] and the CLM processes have been added. Blue arrows indicate water fluxes; yellow arrows indicate short-wave radiation fluxes; brown arrows with outlines indicate longwave radiation; orange arrows indicate heat fluxes; grey arrows indicate carbon fluxes and green arrows are nitrogen fluxes. Abbreviations: $E_{D}$ : evaporation from depression storage; $E_{0}$ : evaporation from channels; $E_{c}$ : canopy evaporation; I: infiltration; $R$ : recharge; $P$ : precipitation; $T$ : transpiration; $T_{c}$ : canopy temperature; $E_{B}$ : evaporation from bare ground; $Q_{\text {thru: }}$ through flow; $Q_{\text {stem }}$ : stemflow; , $N_{\text {dep }}$ : nitrogen deposition; SW: short-wave radiation; $\mathrm{LW}$ : long-wave radiation; $\mathrm{SH}$ : sensible heat. Carbon and nitrogen fluxes were not annotated to avoid clutter. Un-annotated terms include carbon storage and allocation, litterfall, soil respiration, mineral nitrogen uptake, denitrification, and leaching. 


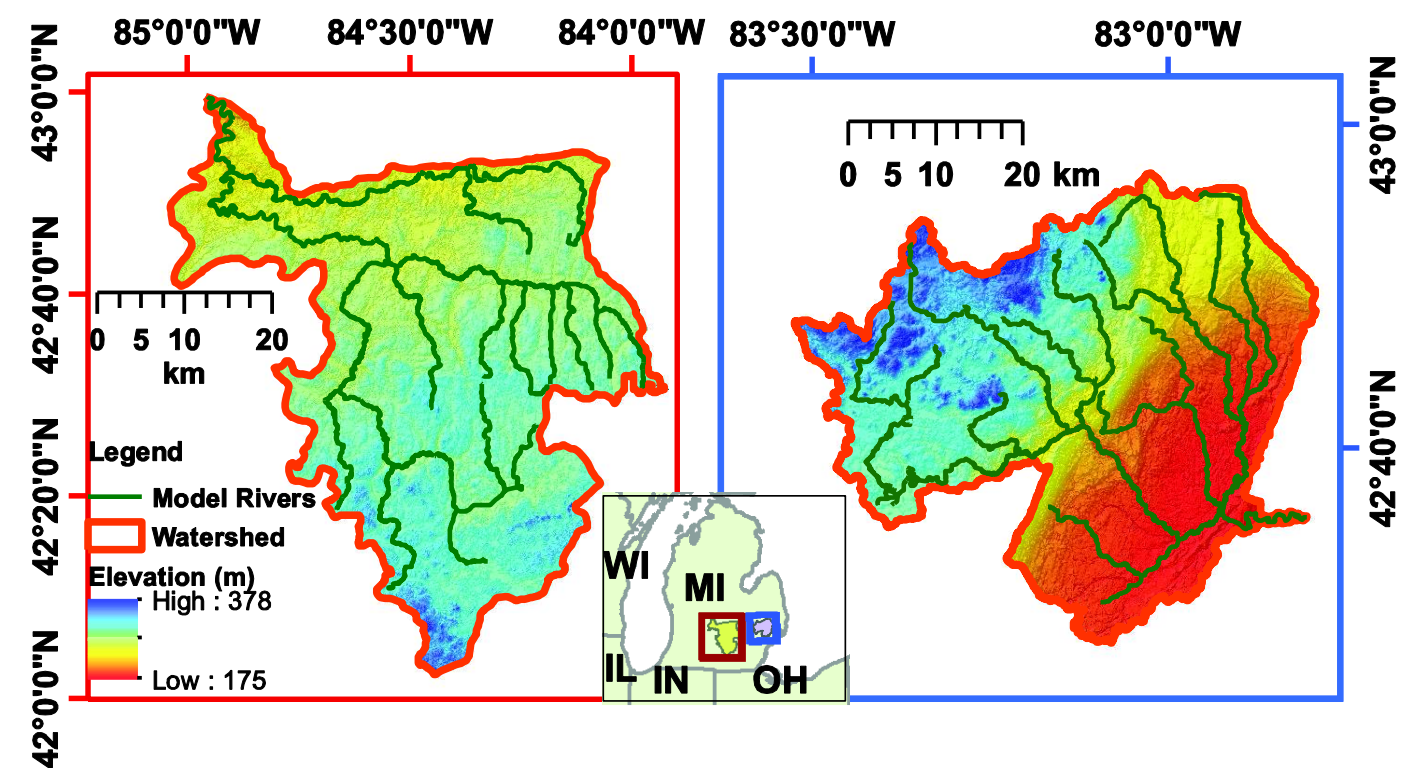

Figure 2. Map of the two basins studied: (a) Upper Grand River Basin (UG) and (b) Clinton River Basin (CRB) in Michigan, USA. The CRB has rugged hills in the western half and a lowland plain in the eastern half, and is adjacent to the Lake St. Clair to the east. The UG has mild relief and lacked the topographical contrast in CRB. 
(a)

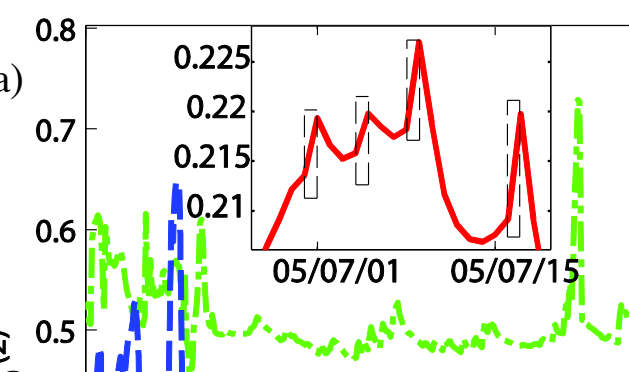

ฮ

$\circ$

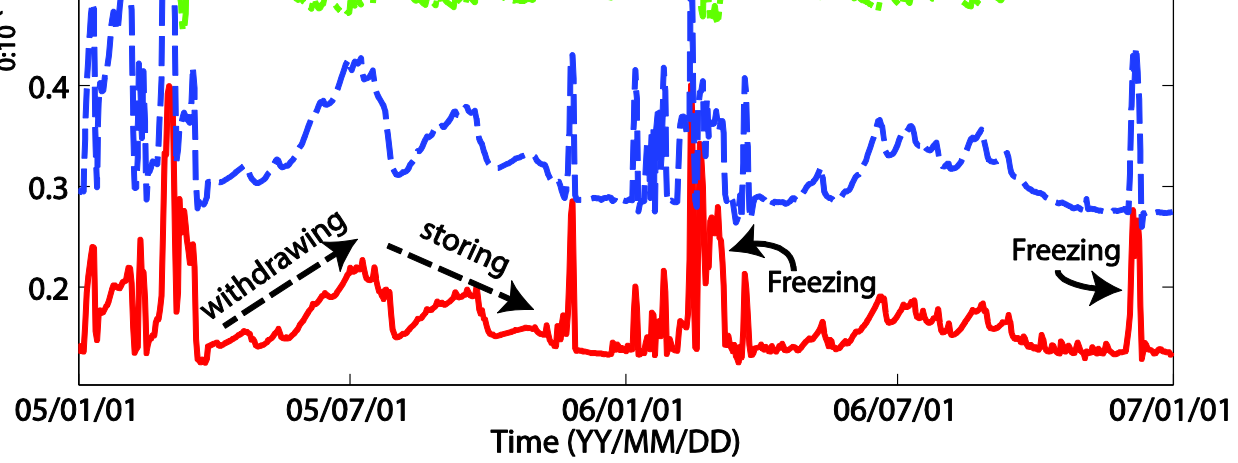

(b)

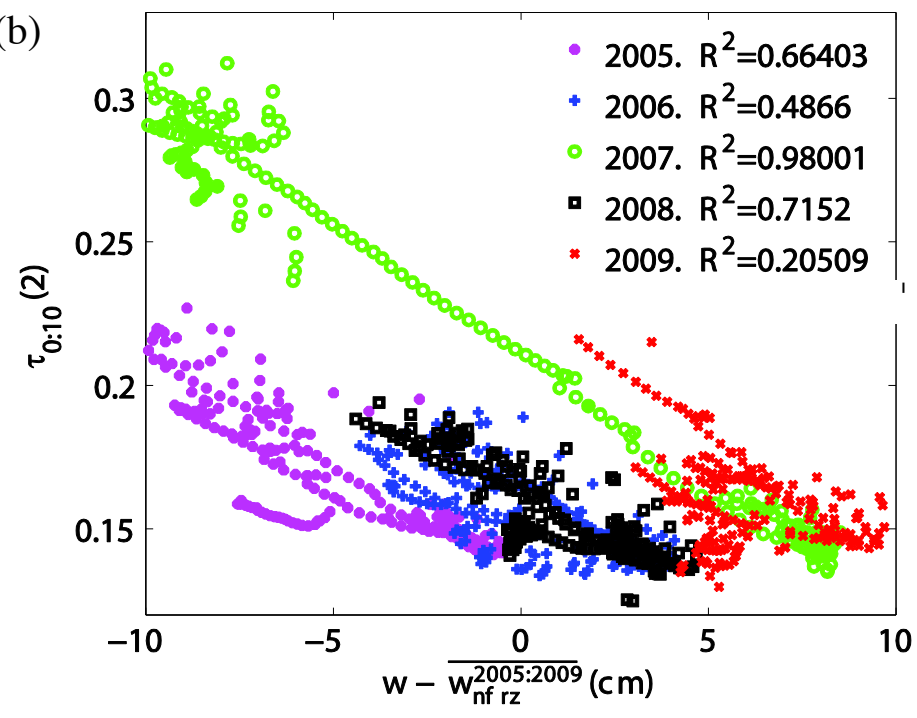

Figure 3. (a) Time evolution of the scaling exponents in the CRB. The seasonal withdrawing and 818 storing limbs and the effects of freezing have been annotated. The subpanel zooms into June, 2005 on the whole basin time series. The dashed boxes indicate days with rainfall; (b) Relationship between $\tau_{0: 10}(2)$ and basin-average water storage anomaly during non-frozen period (April - Oct) in 2005-2009. 

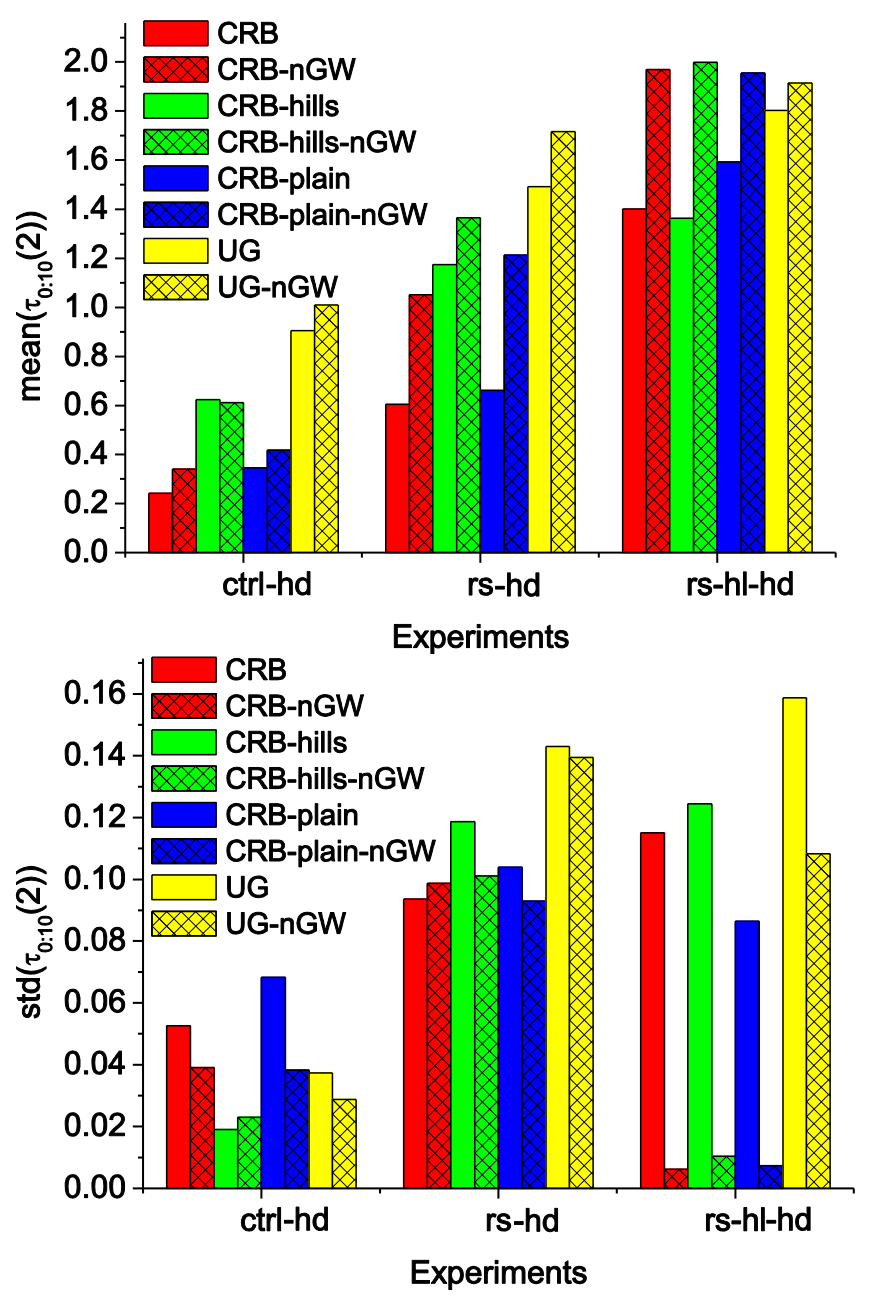

828 Figure 4. (a) temporal mean and (b) temporal standard deviation of $\boldsymbol{\tau}_{0: 10}(2)$ as a function of 829 controls. The colors indicate domains. The solid-filled bars are groundwater enabled simulations $830(\boldsymbol{G W})$ while the hatched bars are groundwater disabled $(n \boldsymbol{G W})$ simulations. 

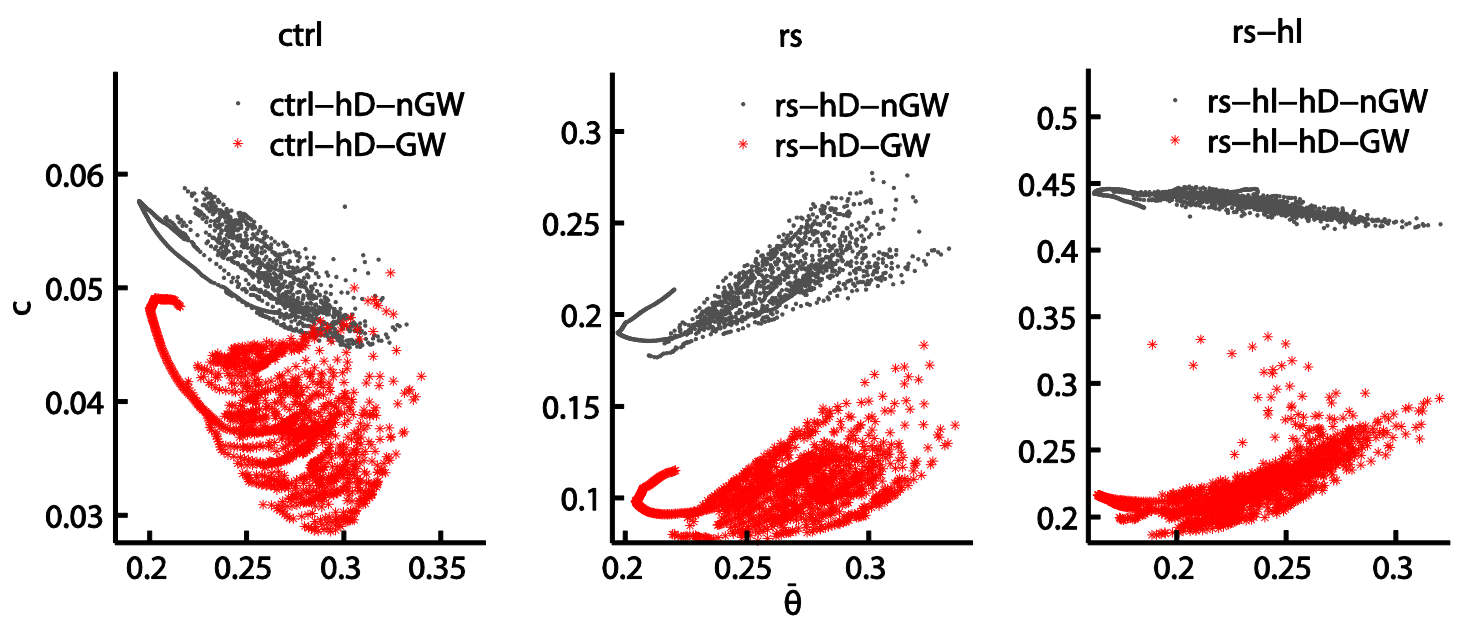

Figure 5. Relationships between $\mathrm{c}$ and $\bar{\theta}$ in the hypothetical simulations. 

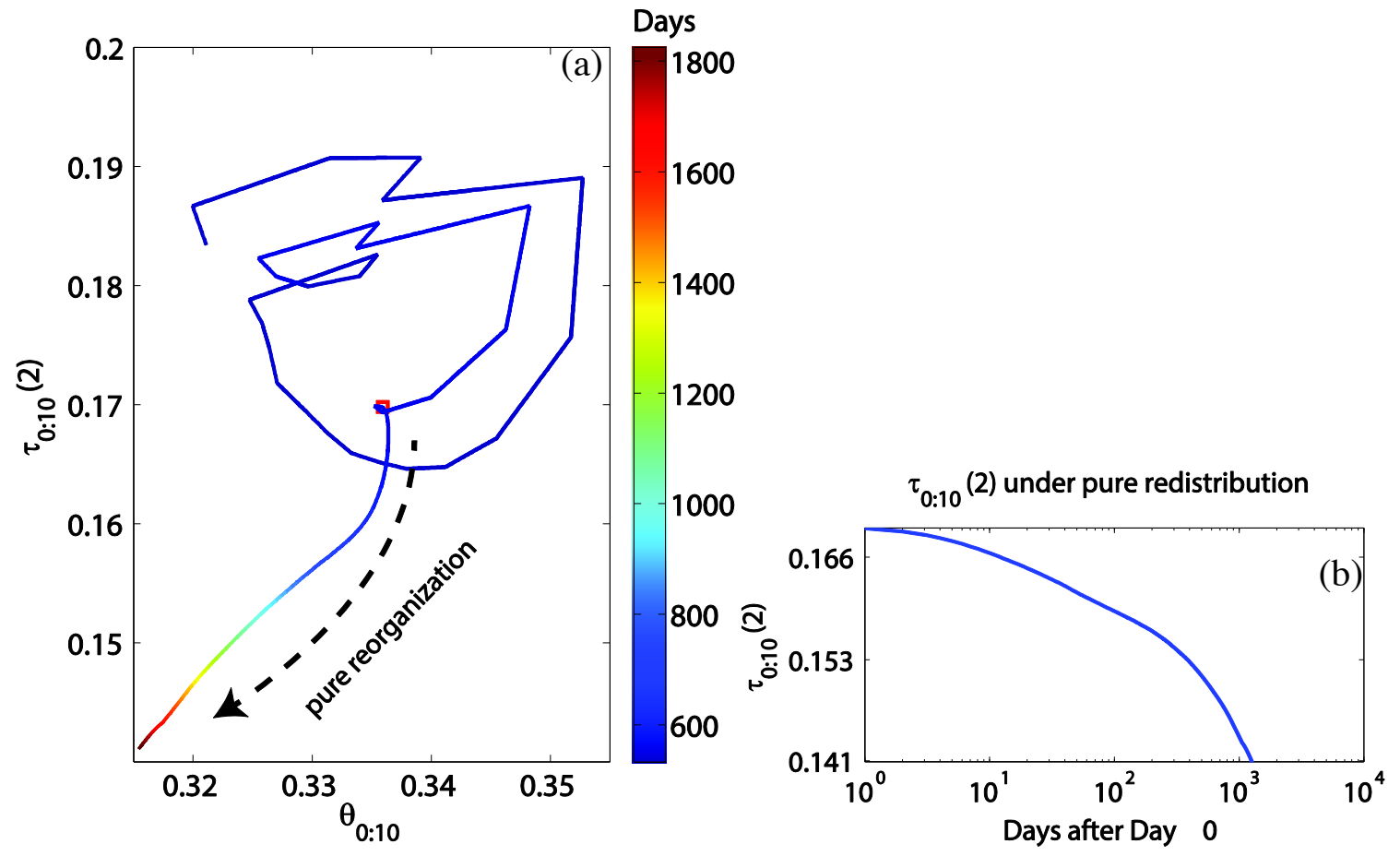

Figure 6. $\tau_{0: 10}(2)$ evolution from noForcing where all water source terms (precipitation and ET) are set to zero on Day 0 (July 16 ${ }^{\text {th }}, 2006$; Julian day 573 of the simulation; red square in (a)) and the basin is subject to pure redistribution. (a) $\tau_{0: 10}(2)$ vs $\theta_{0: 10} ;$ (b) $\tau_{10}^{2}$ vs. time; 

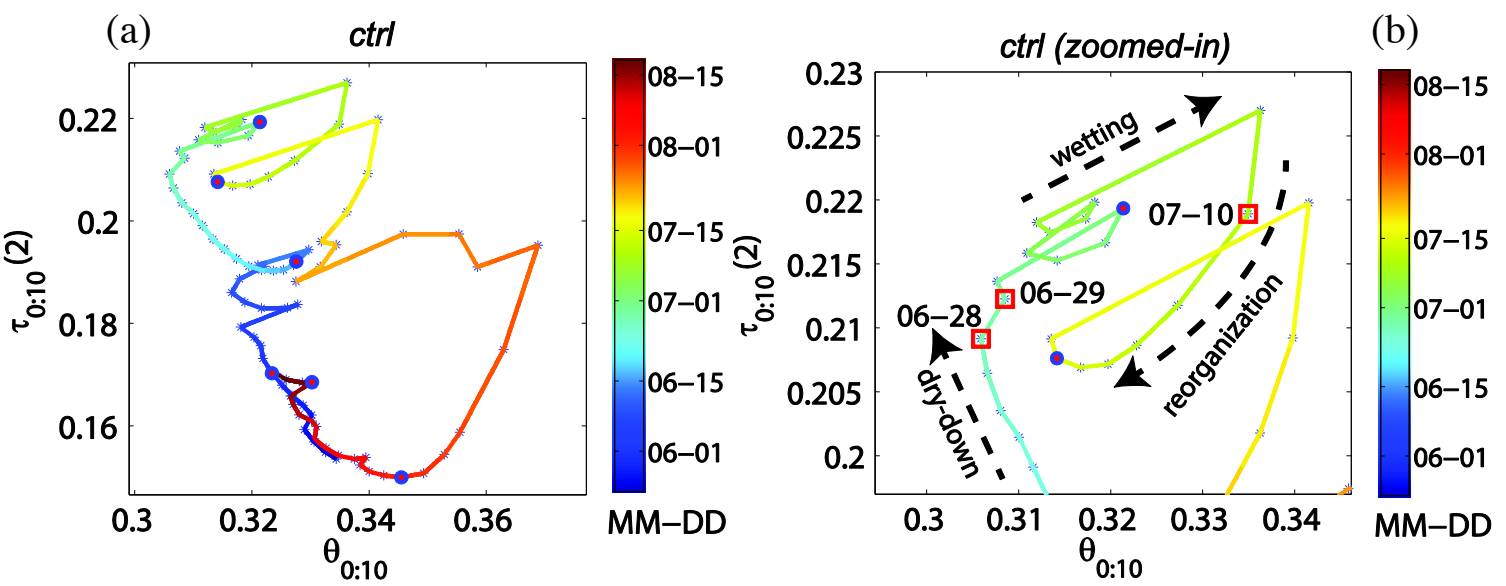

(c)

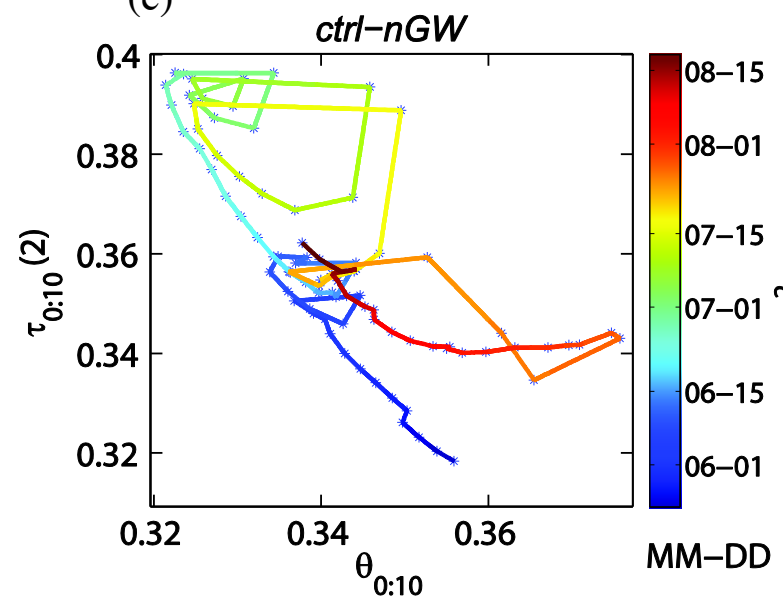

(d)

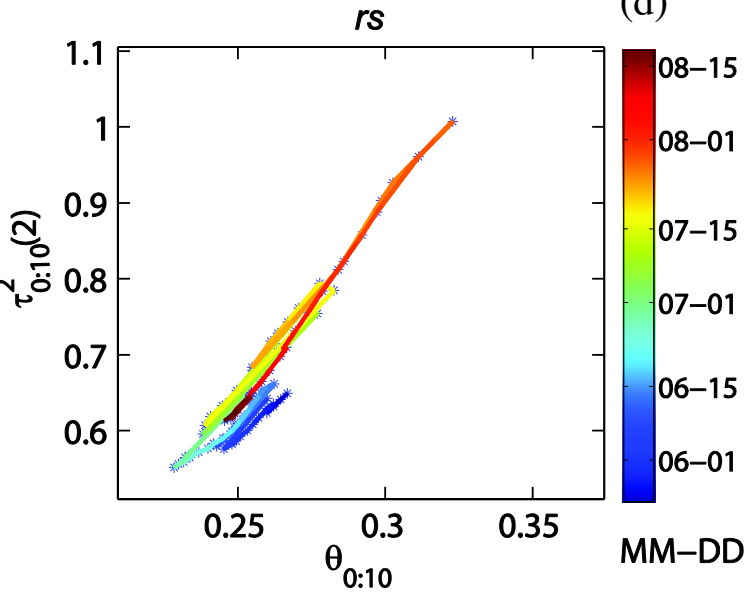

Figure 7. (a) $\tau_{0: 10}(2)$ vs. $\theta_{0: 10}$ (top $10 \mathrm{~cm}$ layer soil moisture) orbits from May $24^{\text {th }}$ to Aug $18^{\text {th }}$, 2005 for the $c t r l$ simulation, with color indicating time; (b) Close-up of the upper half region of (a). The three phases of rainfall-induced excursion have been annotated. The red squares in (b) mark out points corresponding to three annotated days (MM-DD). Color of the lines in all panels indicates time (MM-DD); (c-d) same as in (a), but for, respectively, the groundwater disabled $(c t r l-n G W)$ case and the reshuffled soil texture simulation $(r s)$. When soil textures were reshuffled (LULC and groundwater flow are both in effect), $\tau_{0: 10}(2)$ vs. $\theta_{0: 10}$ relationship changed to positive correlation with little hysteresis, indicating that the patchiness of soil is the primary contributor to the hysteresis and inverse $\tau_{0: 10}(2)$ vs. $\theta_{0: 10}$ relationships observed in the field. 

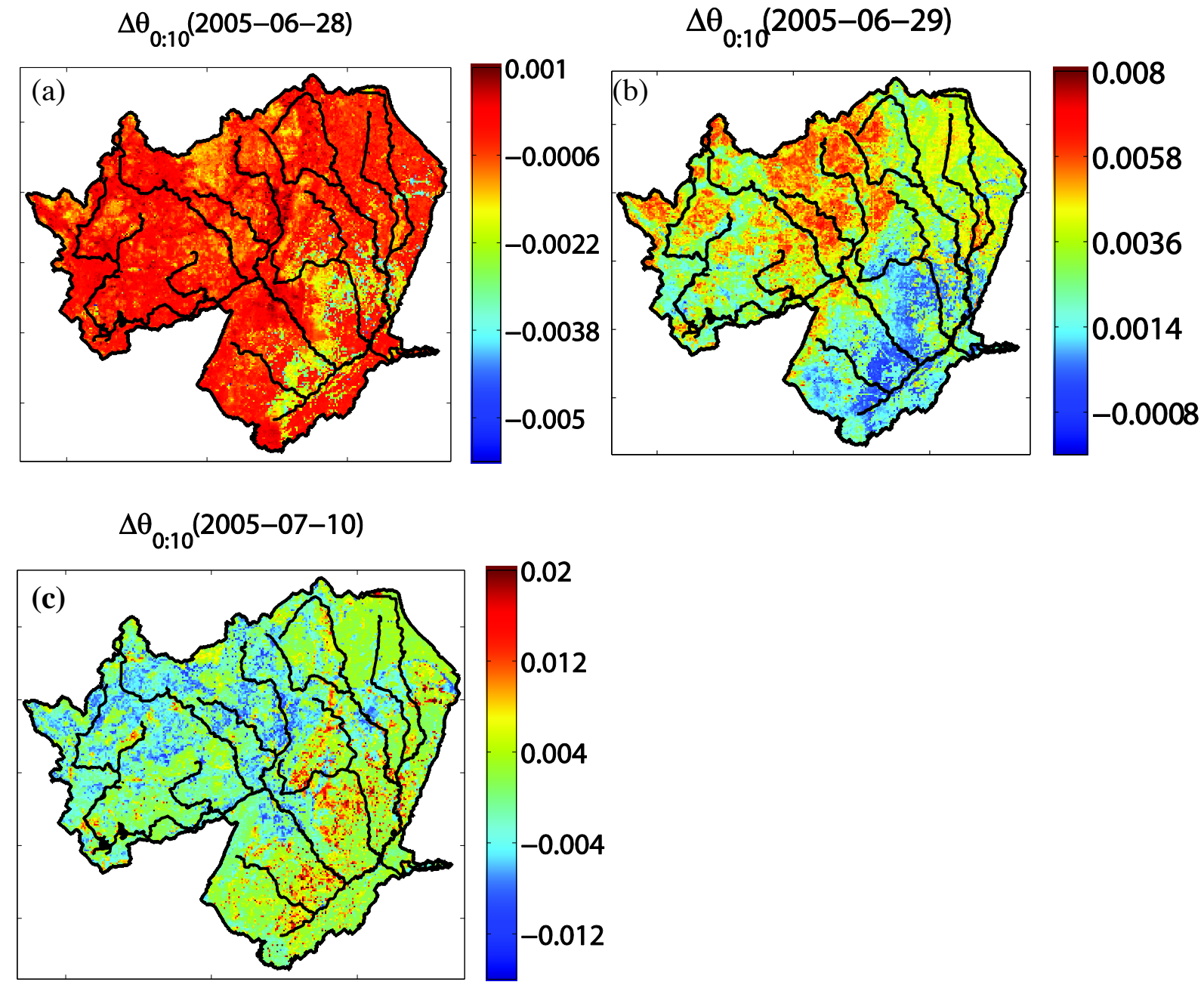

871 Figure 8. $\Delta \theta=\theta(t)-\theta(t-1)$ on selected days in 2005 (these days are marked out by red 872 squares on Figure 7c) from $\mathrm{ctrl}$ : (a) 06-28: last day of a dry-down period; (b) 06-29: a small 873 precipitation event $(3.56 \mathrm{~mm})$; (c) 07-10: first dry day after a large precipitation event. 


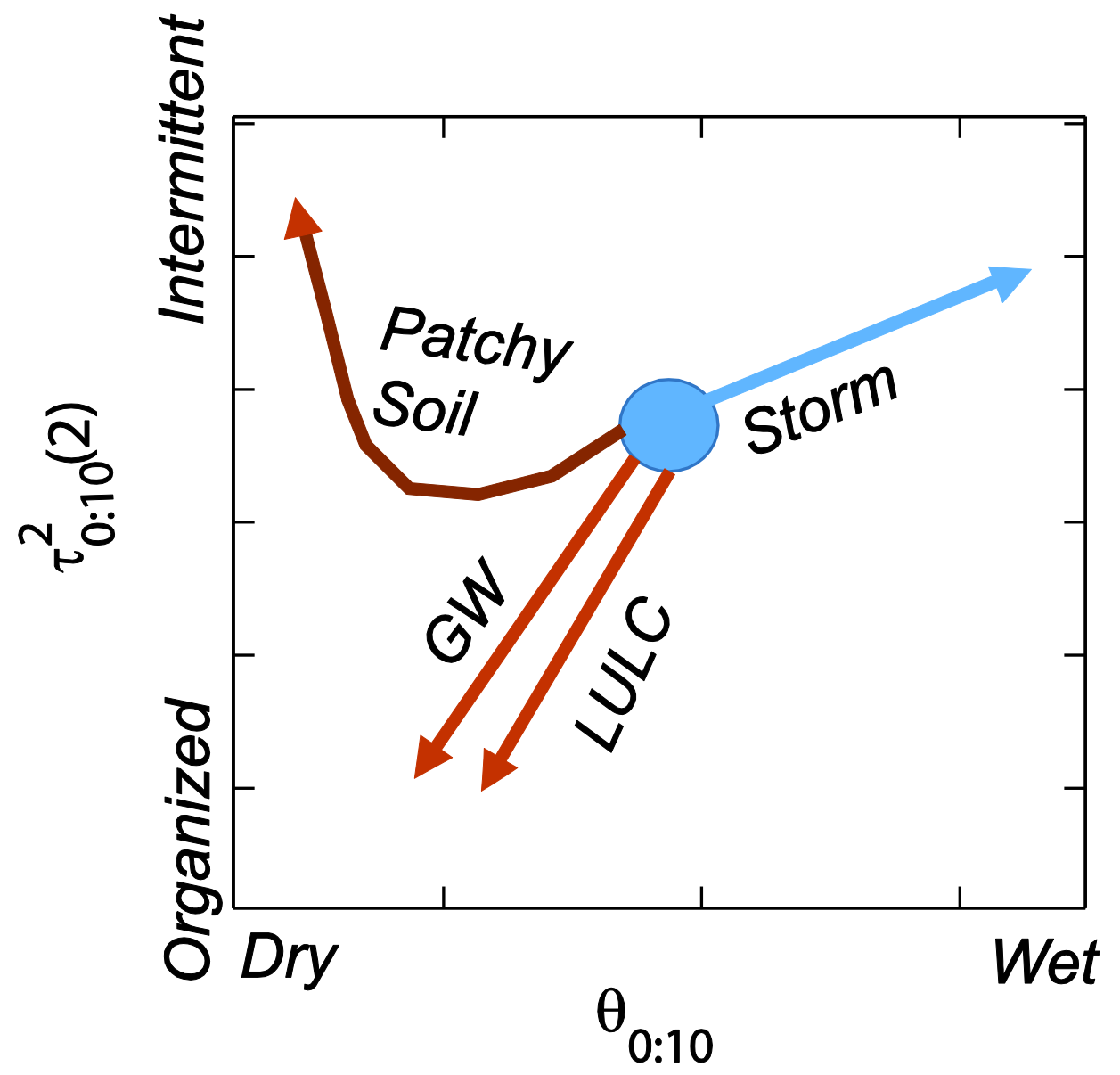

Figure 9. Phenomenological behaviors of different competing processes acting on $\tau_{0: 10}(2)$ : the blue circle indicates initial state. The blue arrow shows the behavior of storm event. The brown arrows show the individual potential effect of groundwater flow (GW), land use land cover (LULC) and spatial aggregation of soil texture (Patchy soil) on fractal evolution during rainfall hiatus following the rainfall. When all factors are present, the system evolves according to the predominant factor (soils), but the effects of other factors are noticeable. 


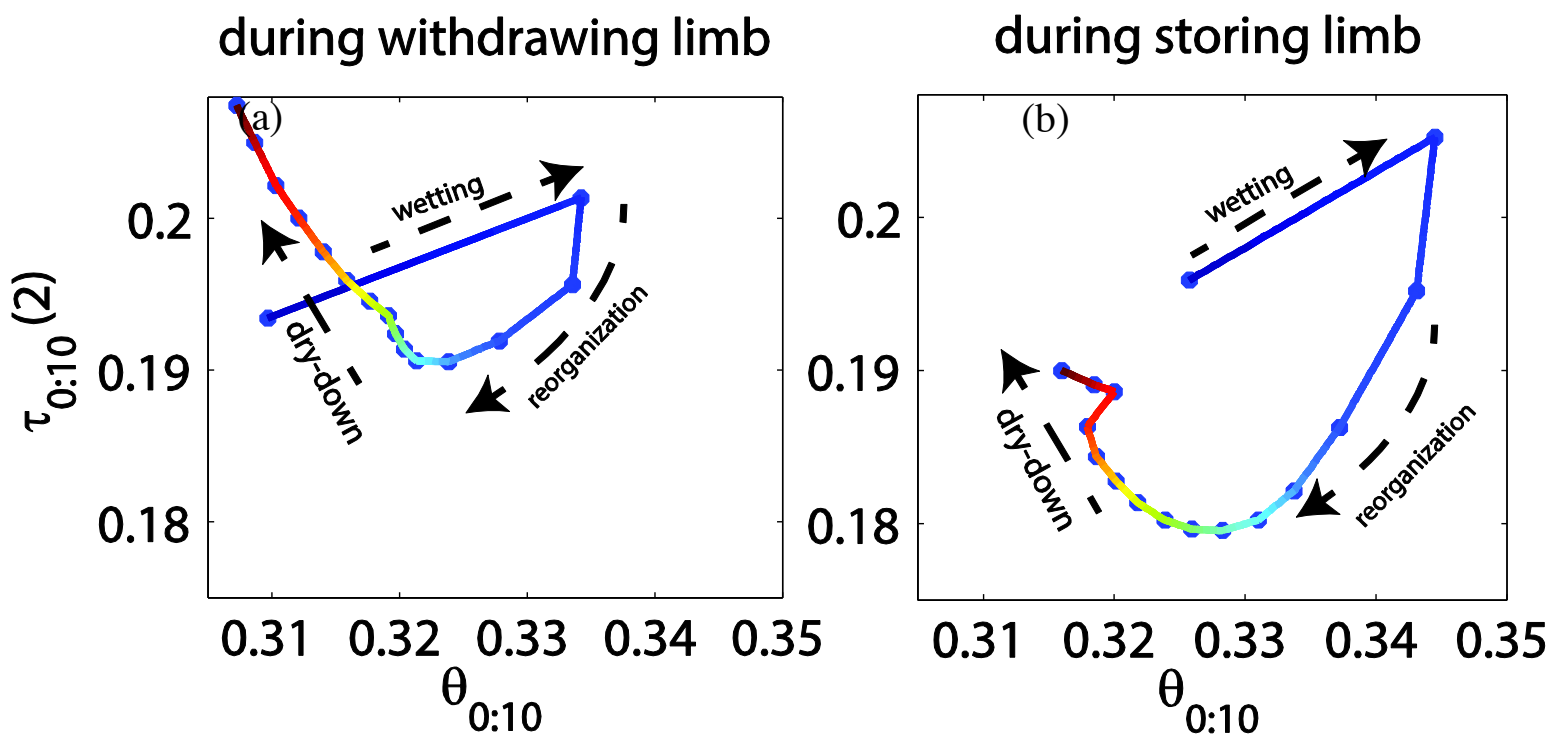

894 Figure 10. Prototypical rainfall-induced excursions during (a) seasonal withdrawing limb and (b) seasonal storing limb. Colors indicate number of days after the last precipitation event and a dot indicates a single day. Both excursions include 17 dry days following the same precipitation amounts $(32 \mathrm{~mm})$ on day 1 . For easy comparison, both panels are set to the same extent on $\boldsymbol{\theta}_{0: 10}$ and $\tau_{0: 10}(2)$ axes. 


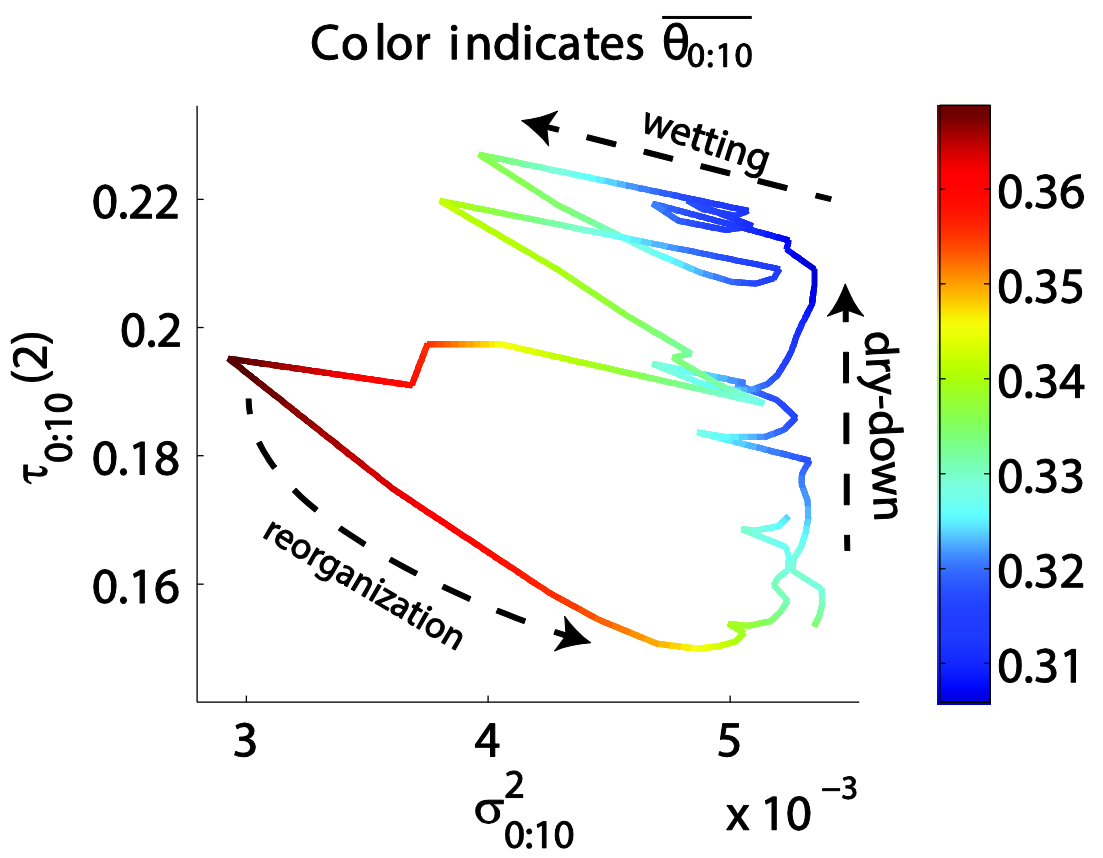

Figure 11. $\tau_{10}^{2}$ vs $\sigma_{10}^{2}$ with color of the line representing the mean moisture from May $24^{\text {th }}$ to Aug 26906 


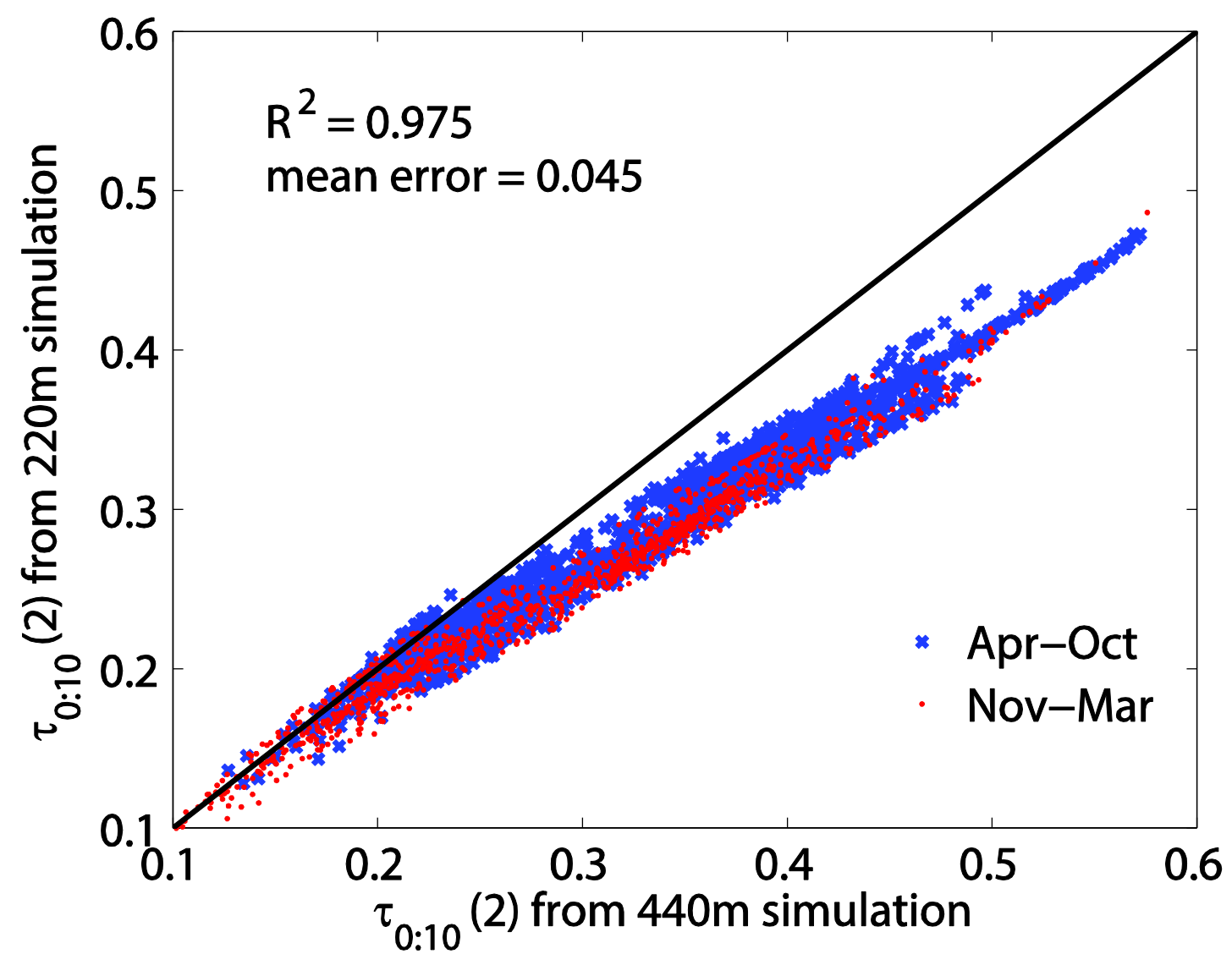

Figure 12. Effect of simulated grid resolution on $\tau_{0: 10}(2)$ from 2002 to 2009. Two groups of points are shown: April to October each year, and November to March next year. 


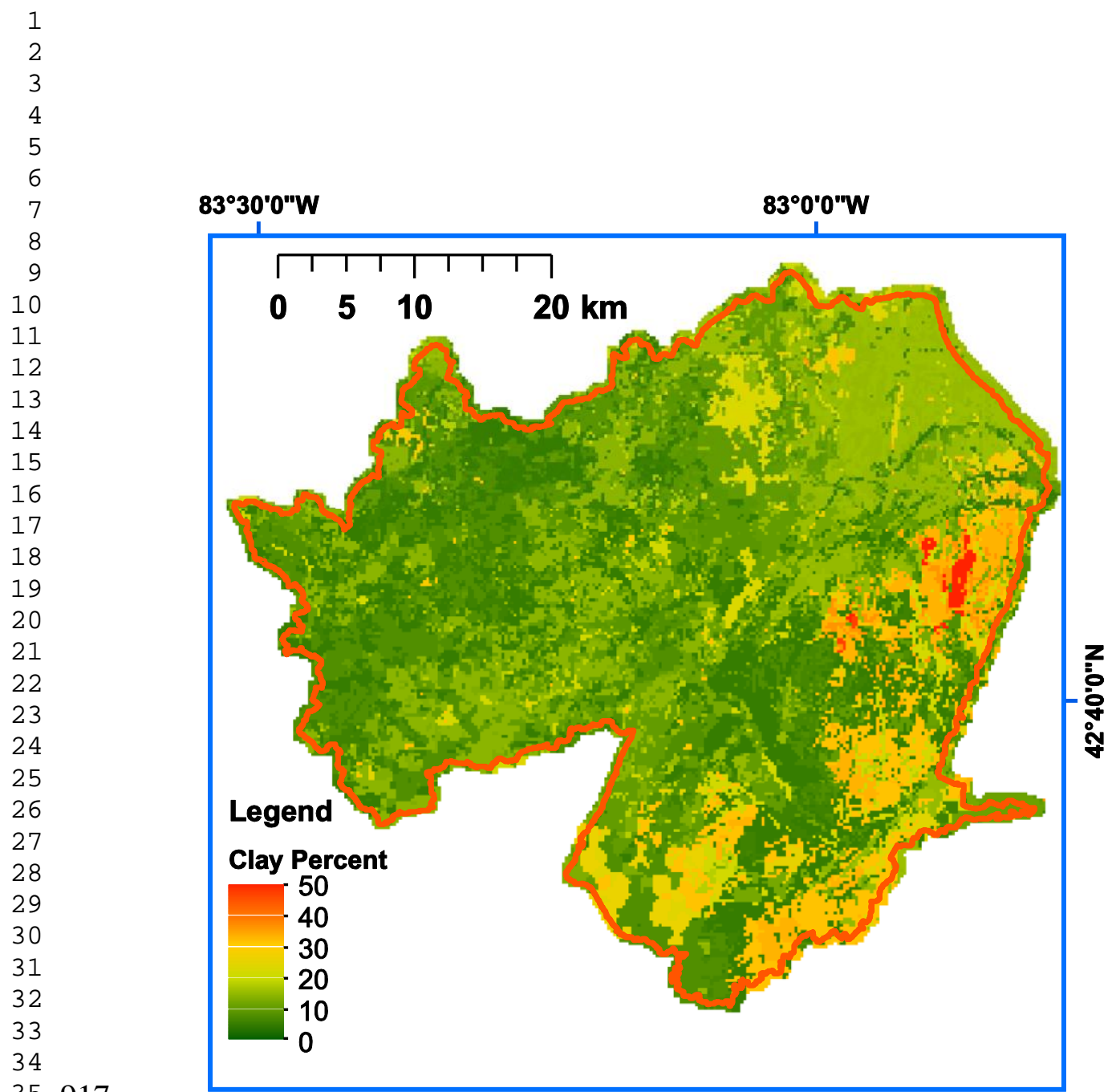

$\begin{array}{ll}35 & 917\end{array}$ 36918 37919

$\begin{array}{ll}38 & 920\end{array}$

40921

41

42

43

44

45

46

47

48

49

50

51

52

53

54

55

56

57

58

59

60

61

62

63

64

65

Figure A1. Map of top-layer clay percentages in the Clinton River basin 
$\theta_{0: 10}(2005-J u n-28)$
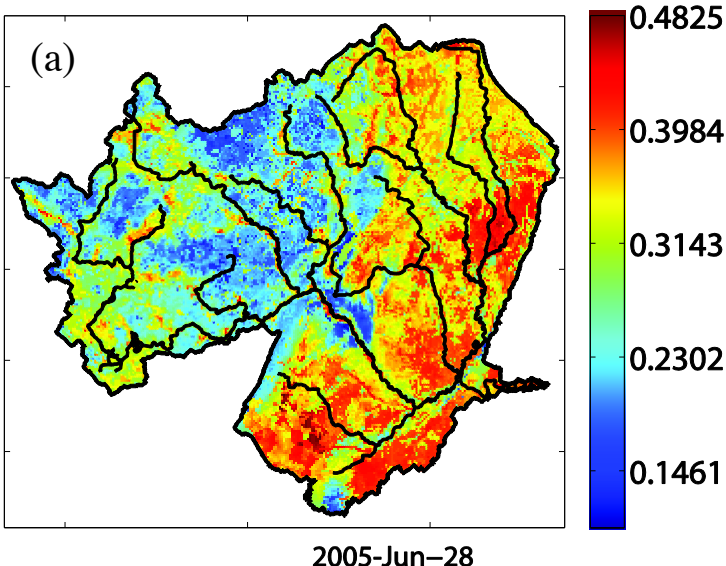

(c)

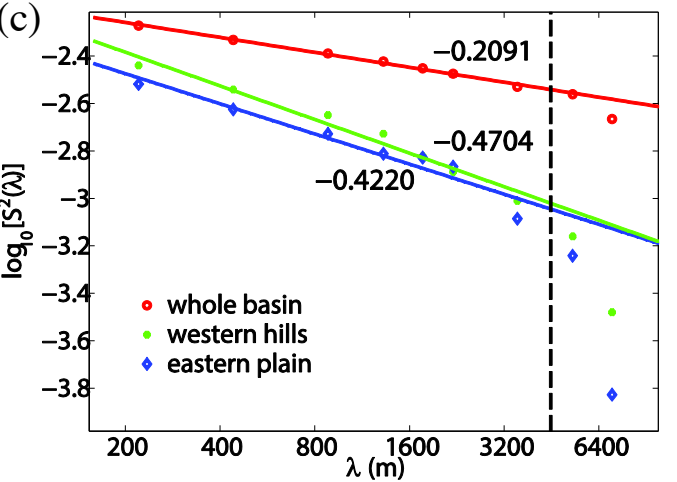

(e)


$\lambda_{\text {max }}=3520$. $\theta_{0: 10}$ (2005-Dec-01)

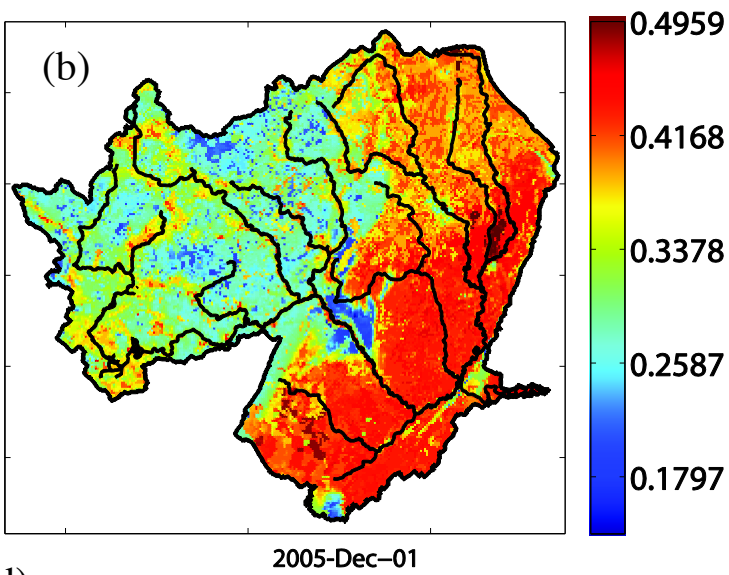

(d)



926 Figure A2. Spatial maps and related scaling of moments for the CRB: (a) $\boldsymbol{\theta}_{0: 10}$ map on 2005-06-28; (b) $\theta_{0: 10}$ map on 2005-12-01; (c) power-law decay of spatial moments in map (a); and (d) power-law decay of spatial moments in map (b). The black dashed lines indicate the fractal cutoff, above which the fractal relationship breaks down. Our estimation of scaling exponents uses only data below the cutoff threshold; (e) detecting nonlinearity using robust regression weights for the points corresponding to $\lambda=3520,5280$ and $7080 \mathrm{~m}$ window sizes, which shows that the $7080 \mathrm{~m}$ point is always above fractal cutoff, $5280 \mathrm{~m}$ point seasonally above cutoff, and $3520 \mathrm{~m}$ point always below the cutoff (never showed statistically significant nonlinearity). Therefore, we choose 


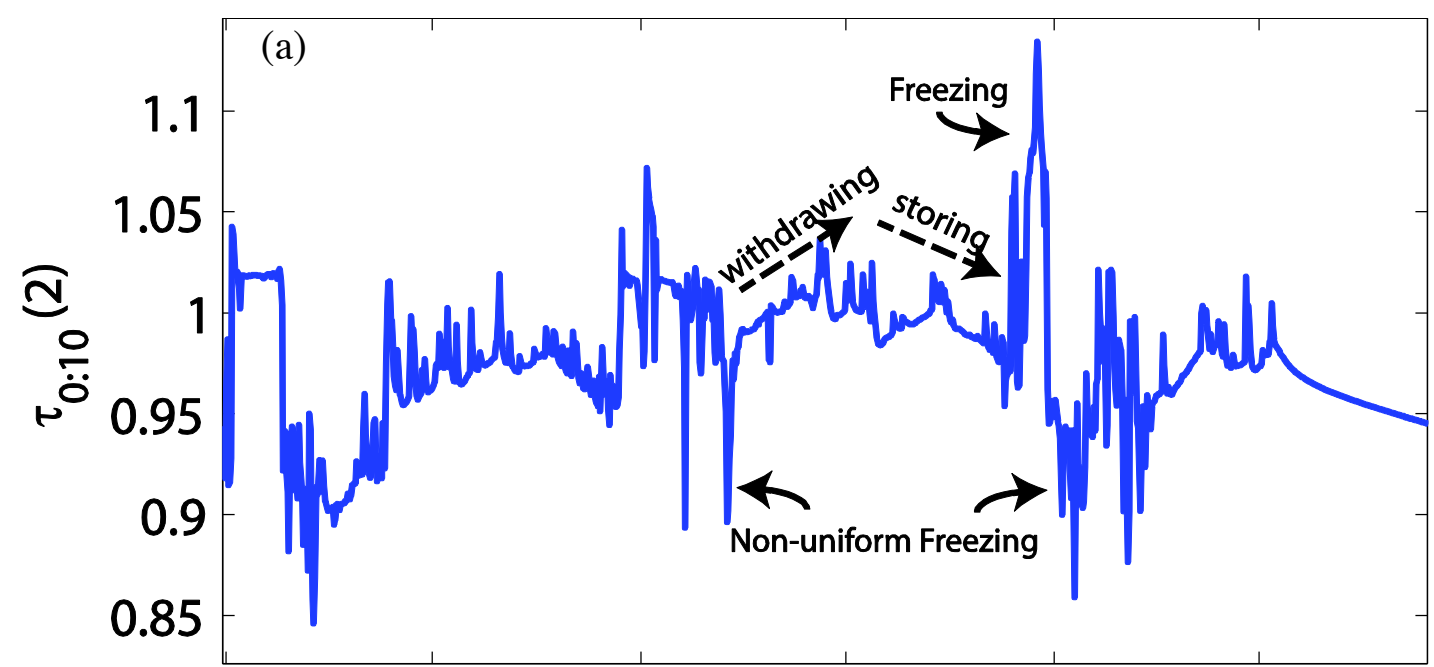
Time (YY/MM)

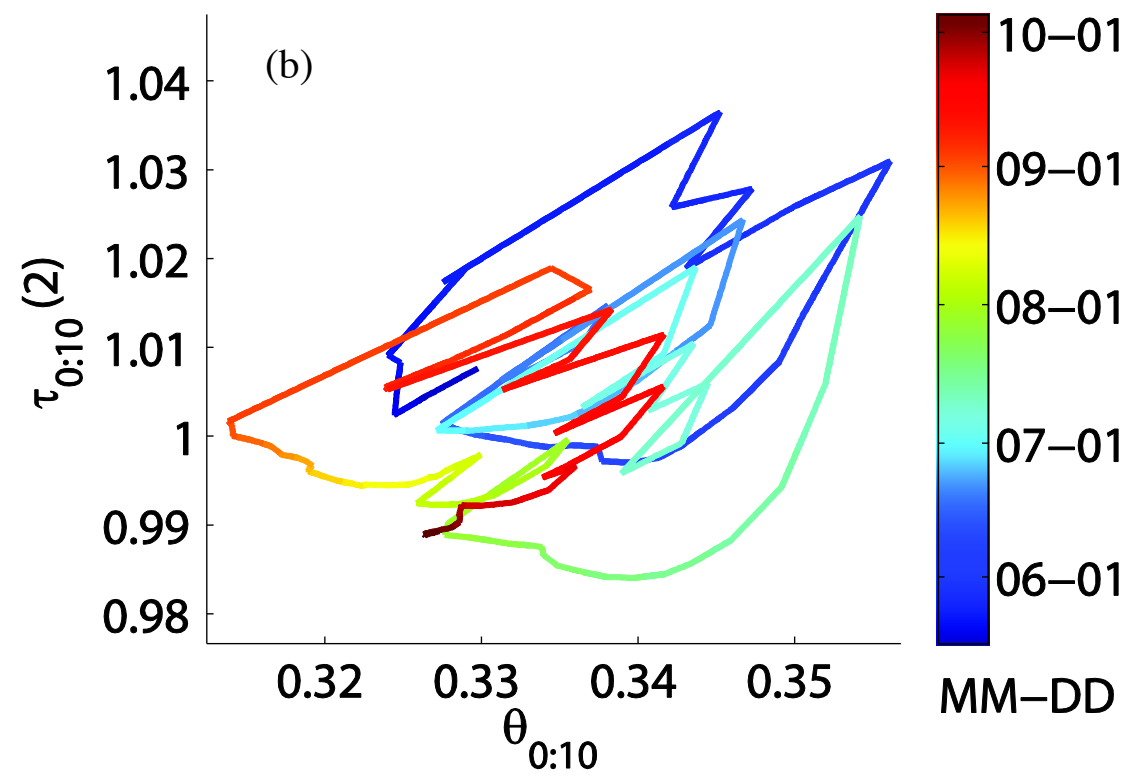

Figure A3. (a) time series of $\tau_{0: 10}(2)$ in the UG in 2004-2006 and (b) $\tau_{0: 10}(2) \sim \theta_{0: 10}$ between May $30^{\text {th }}$ and Oct $18^{\text {th }}, 2005$. Similar to the CRB, we notice seasonal withdrawing and storing limbs, and rainfall-induced excursions, although the range of $\tau_{0: 10}(2)$ is smaller with the UG. 


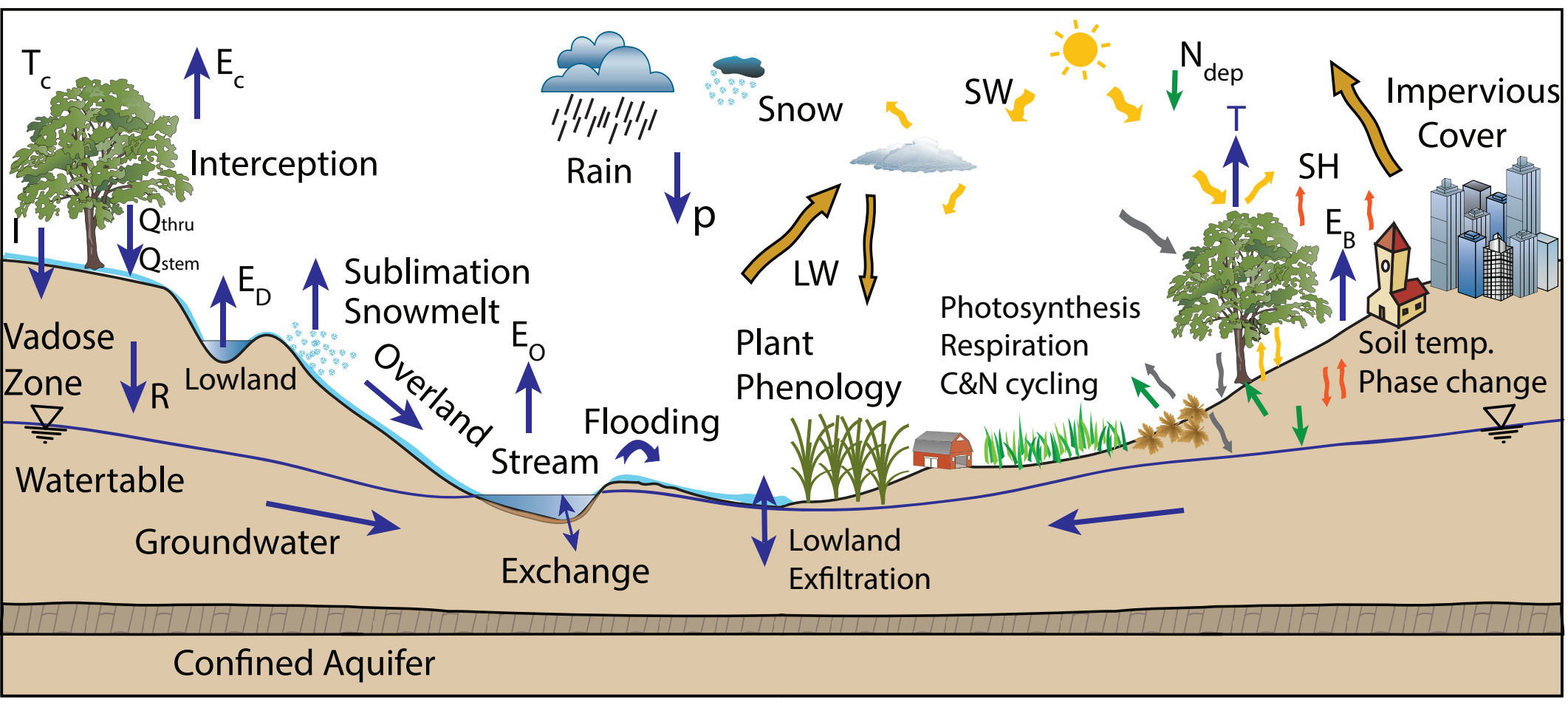




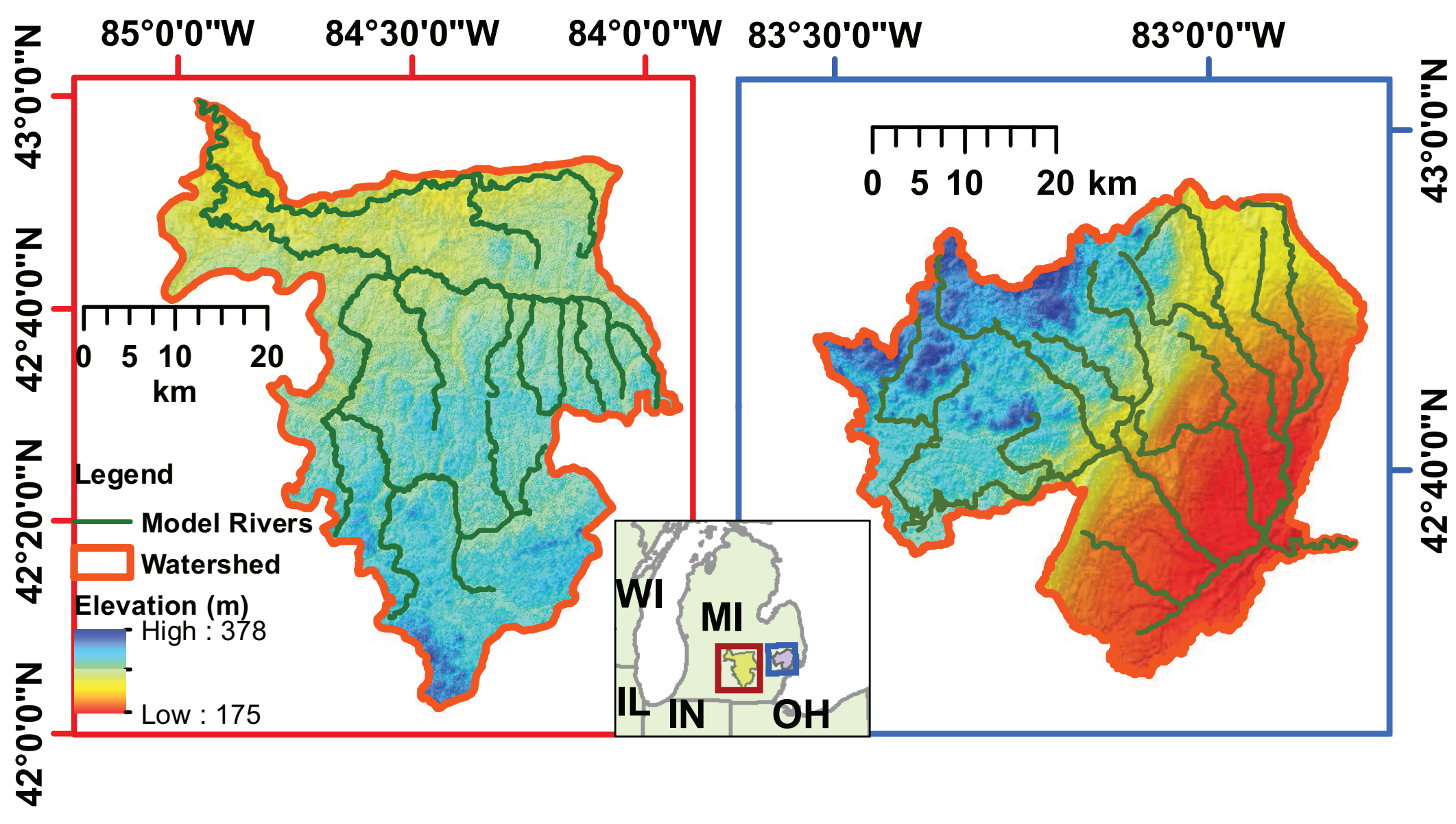




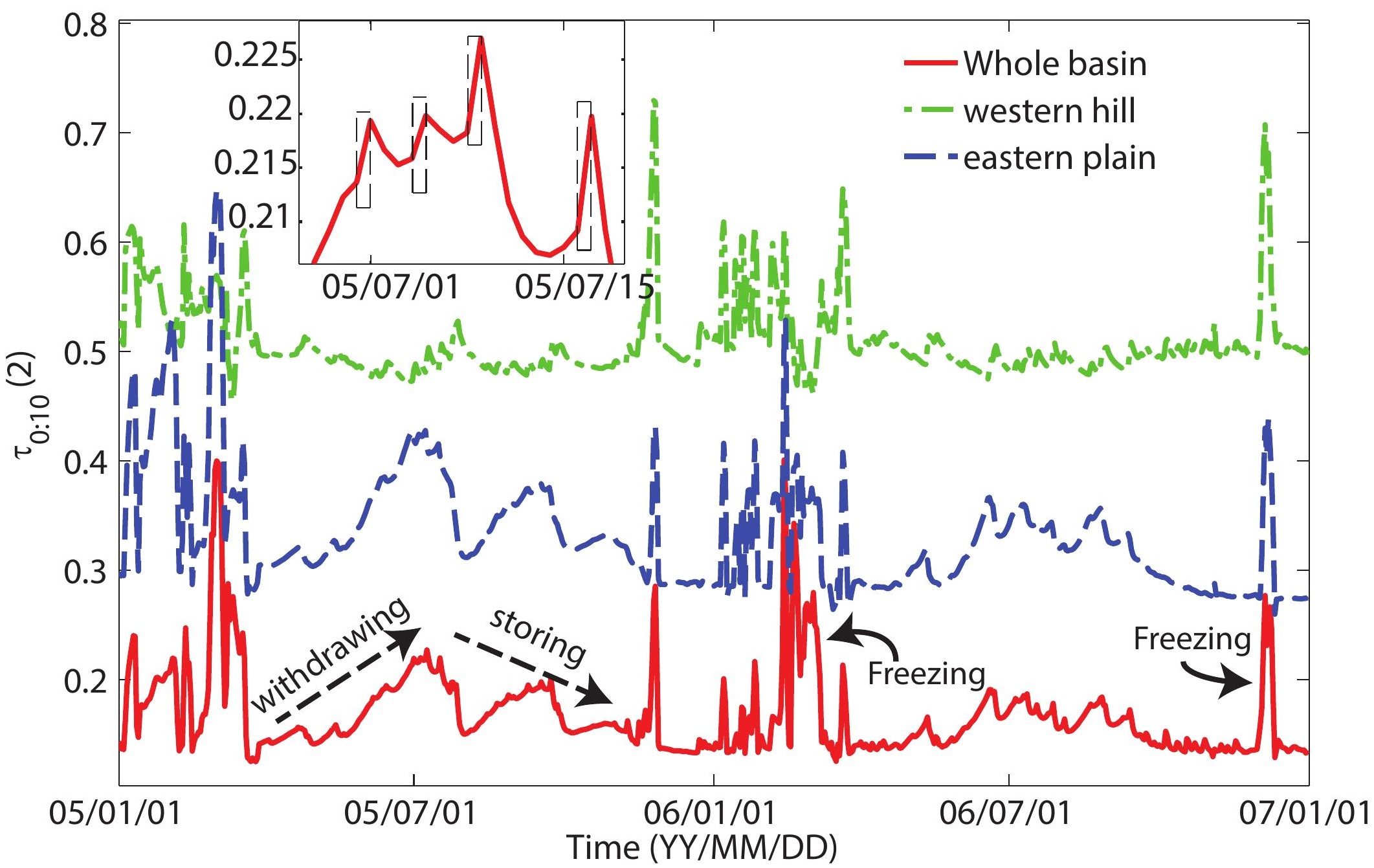




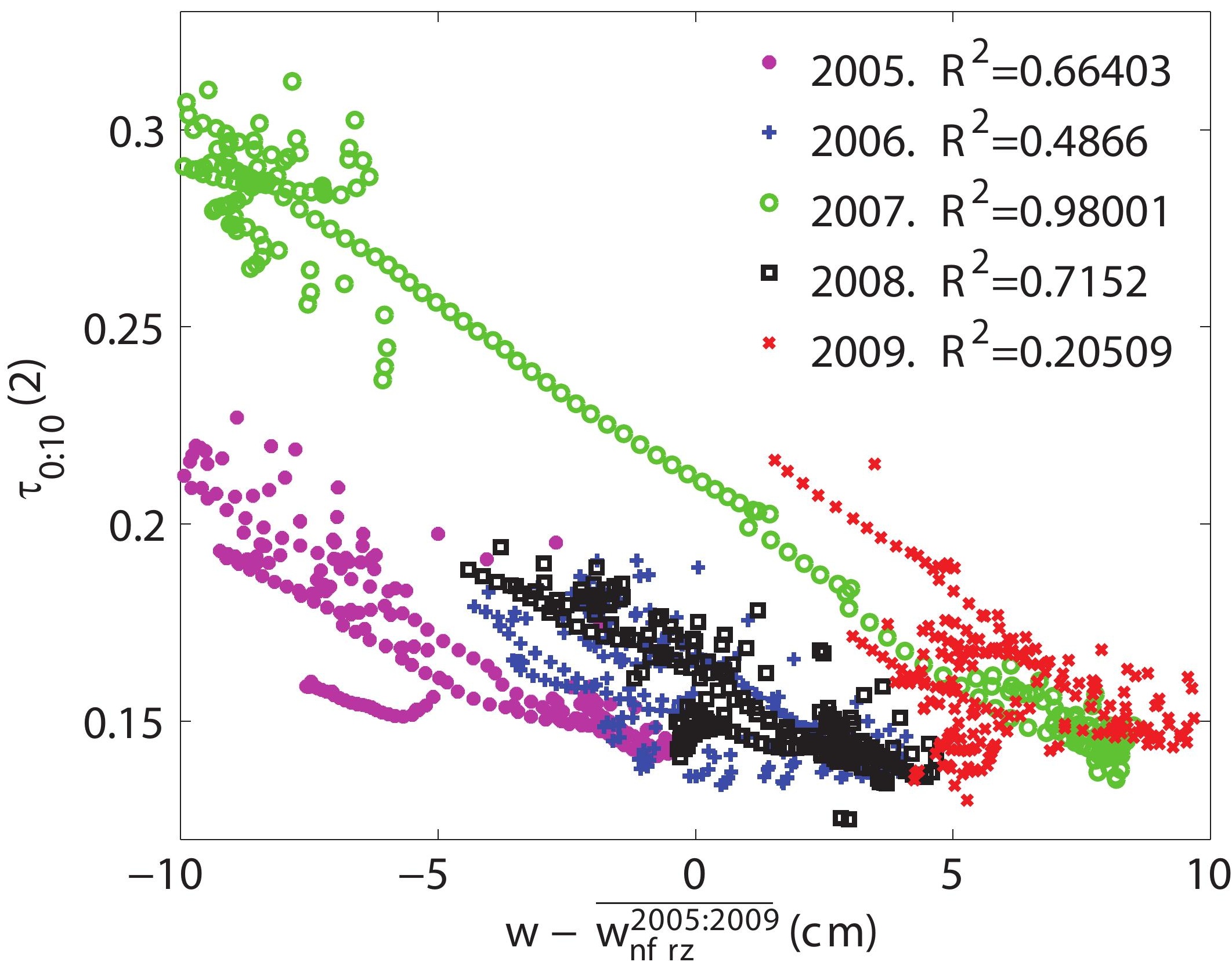




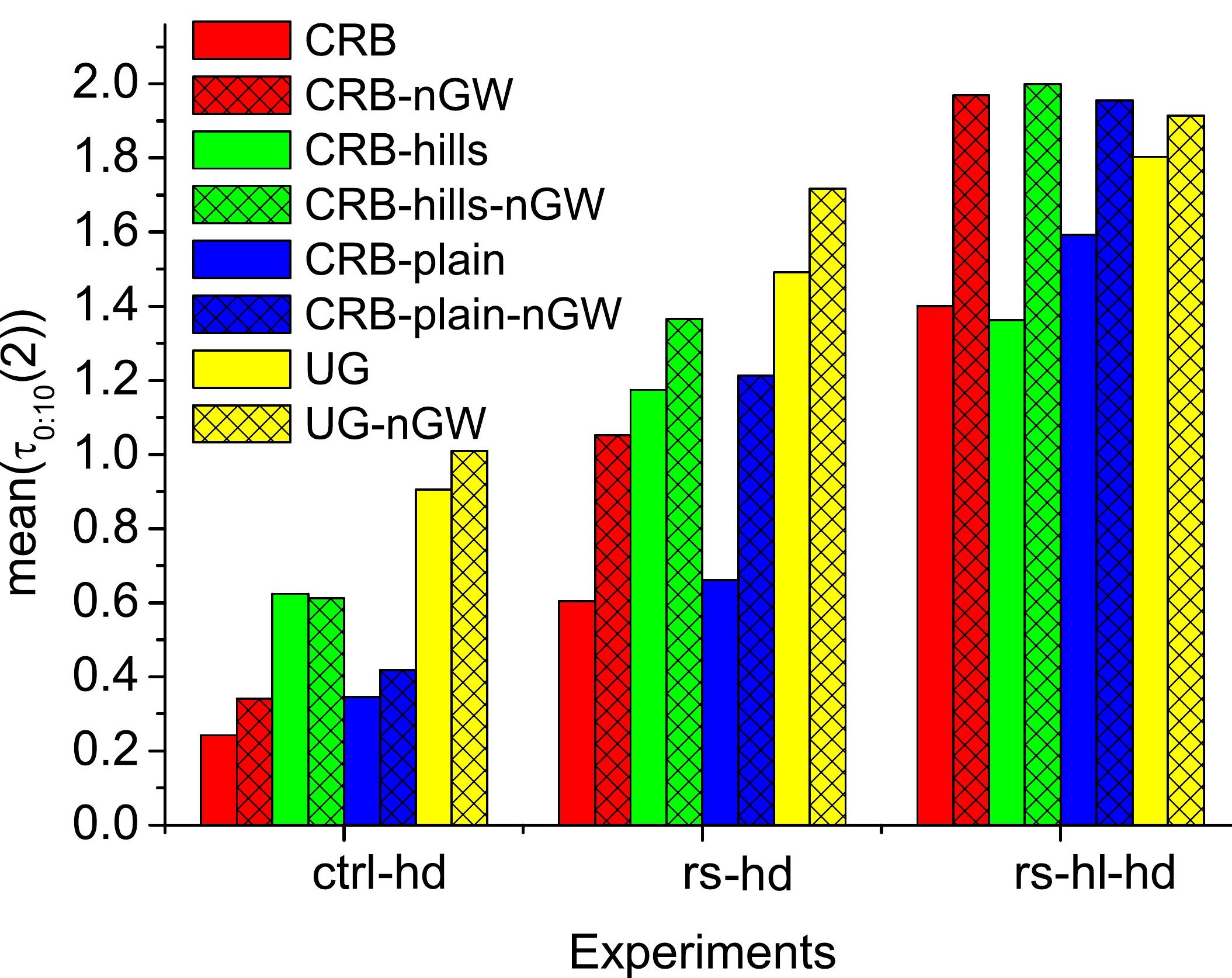




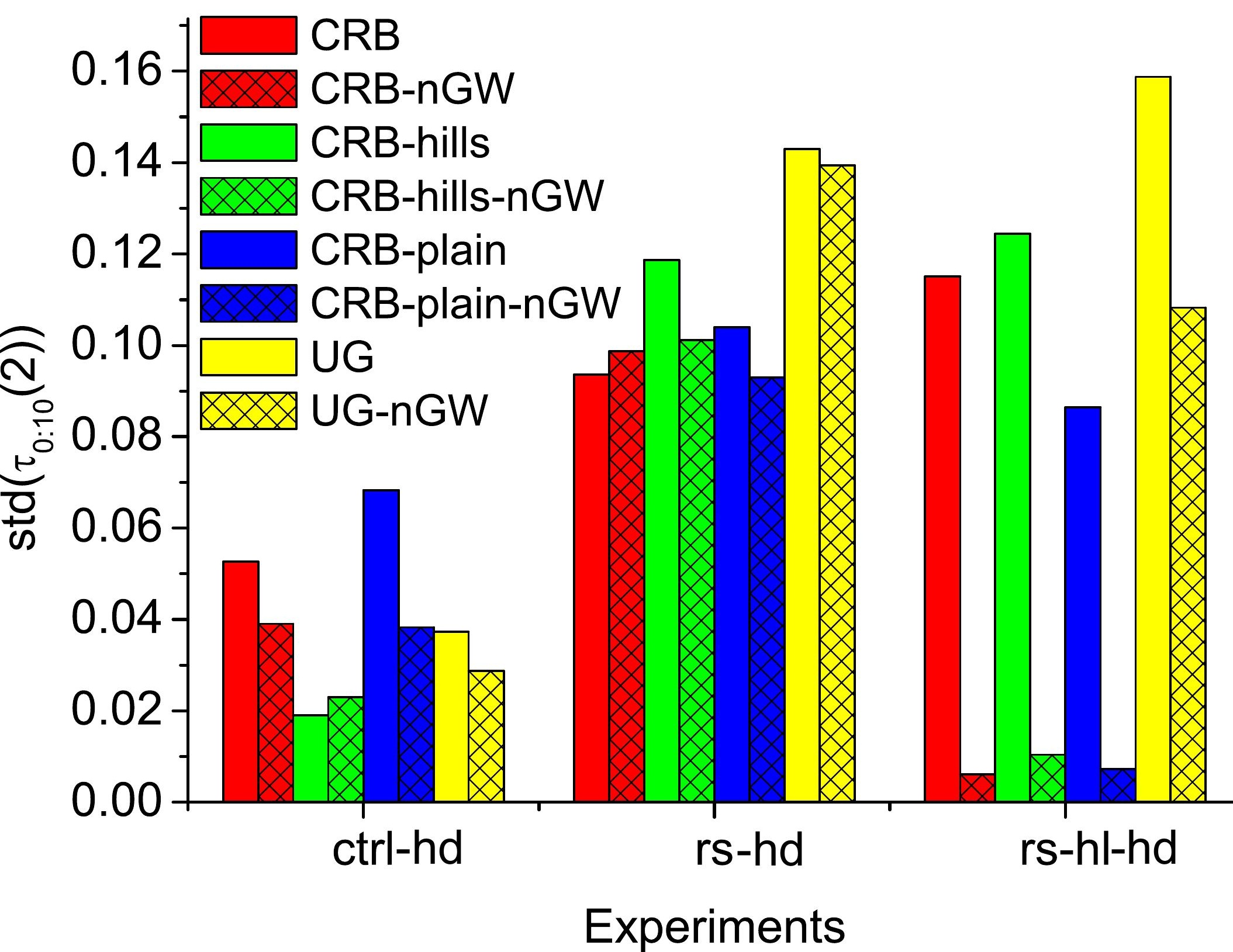



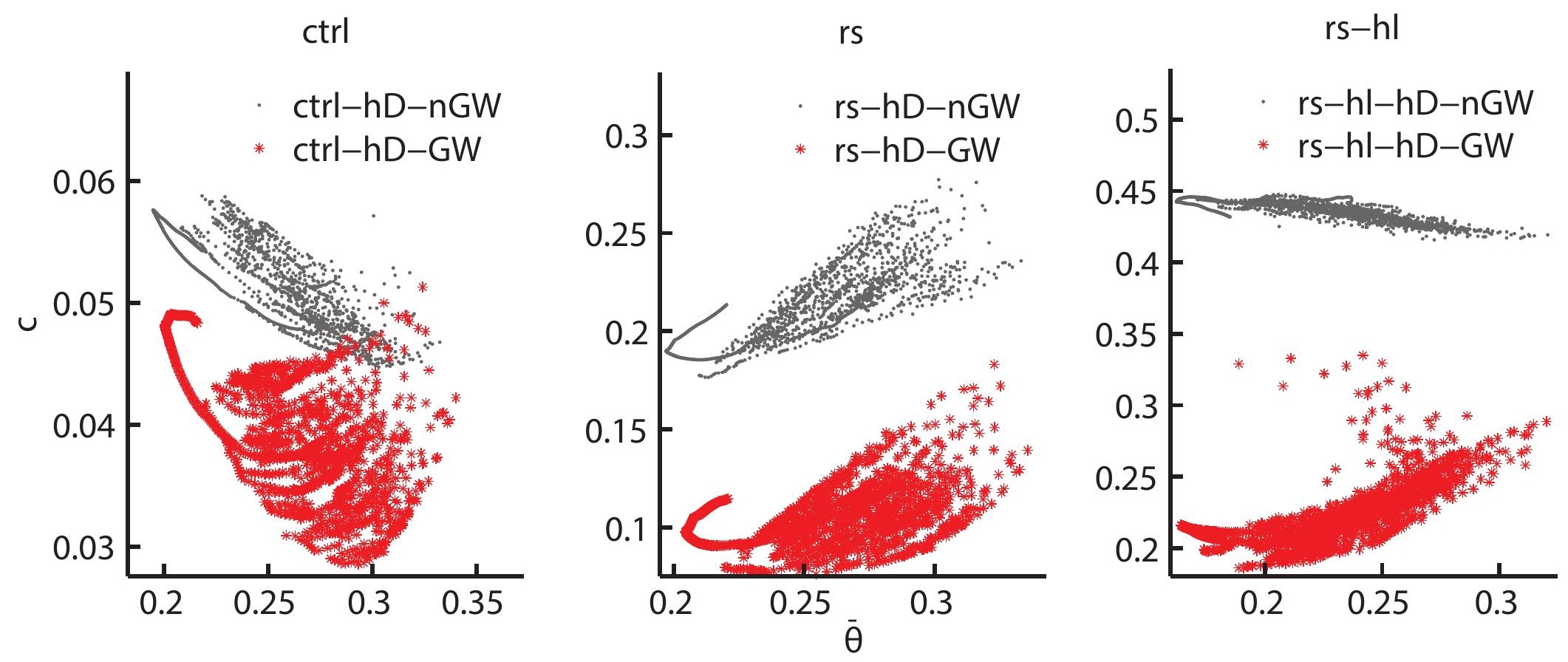




Days

1800

1600

1200

1000

800

600

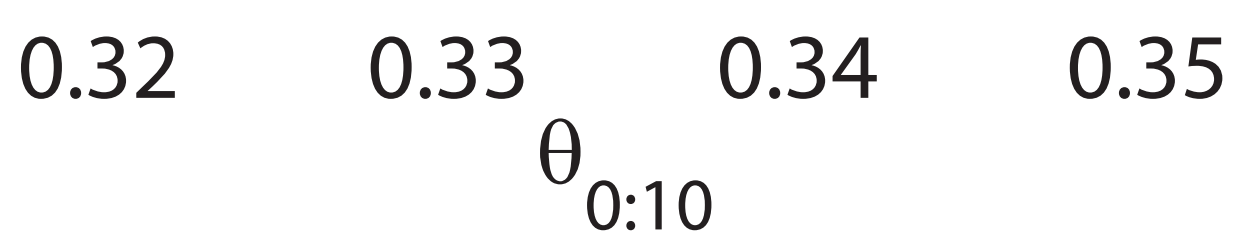


ctrl
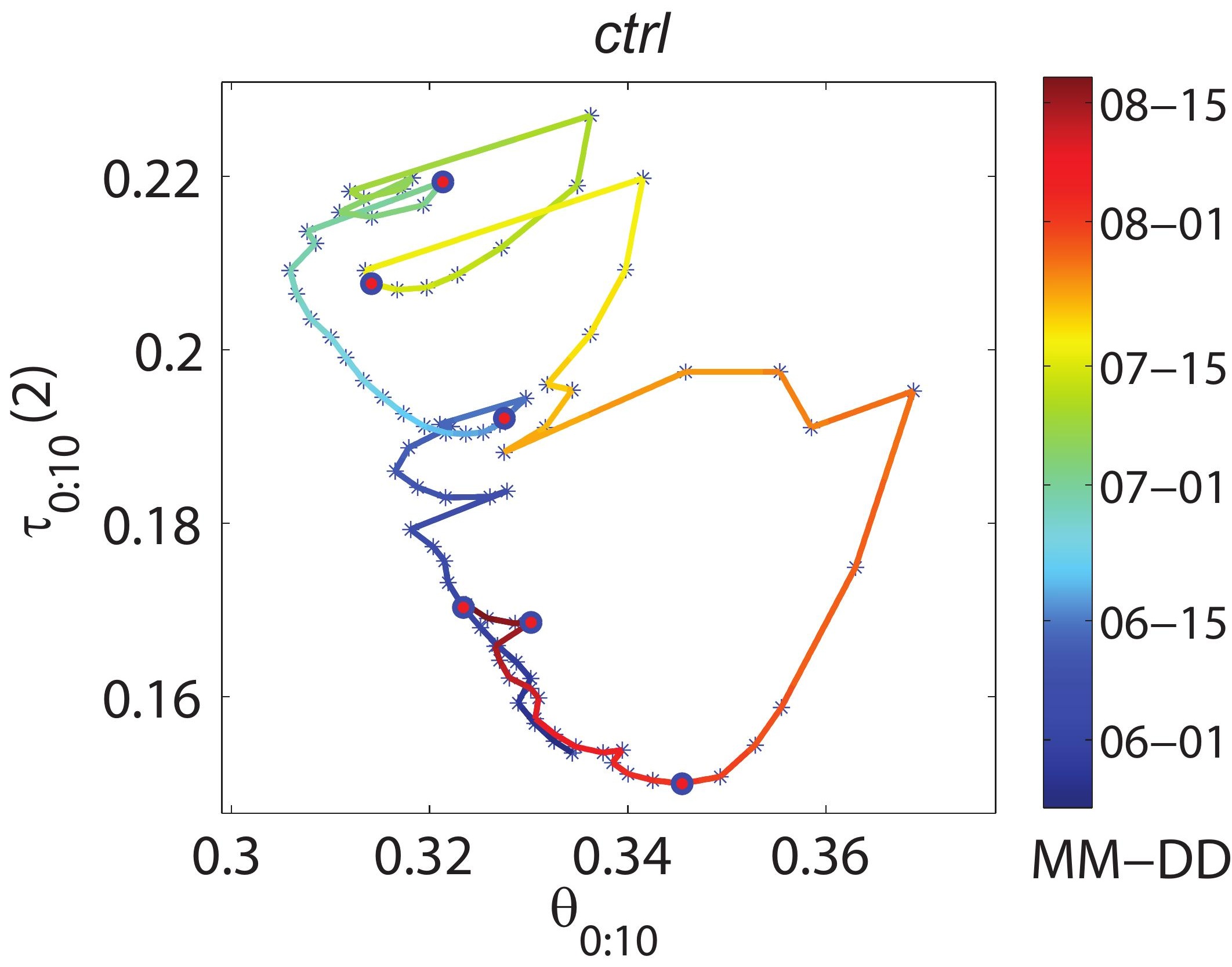


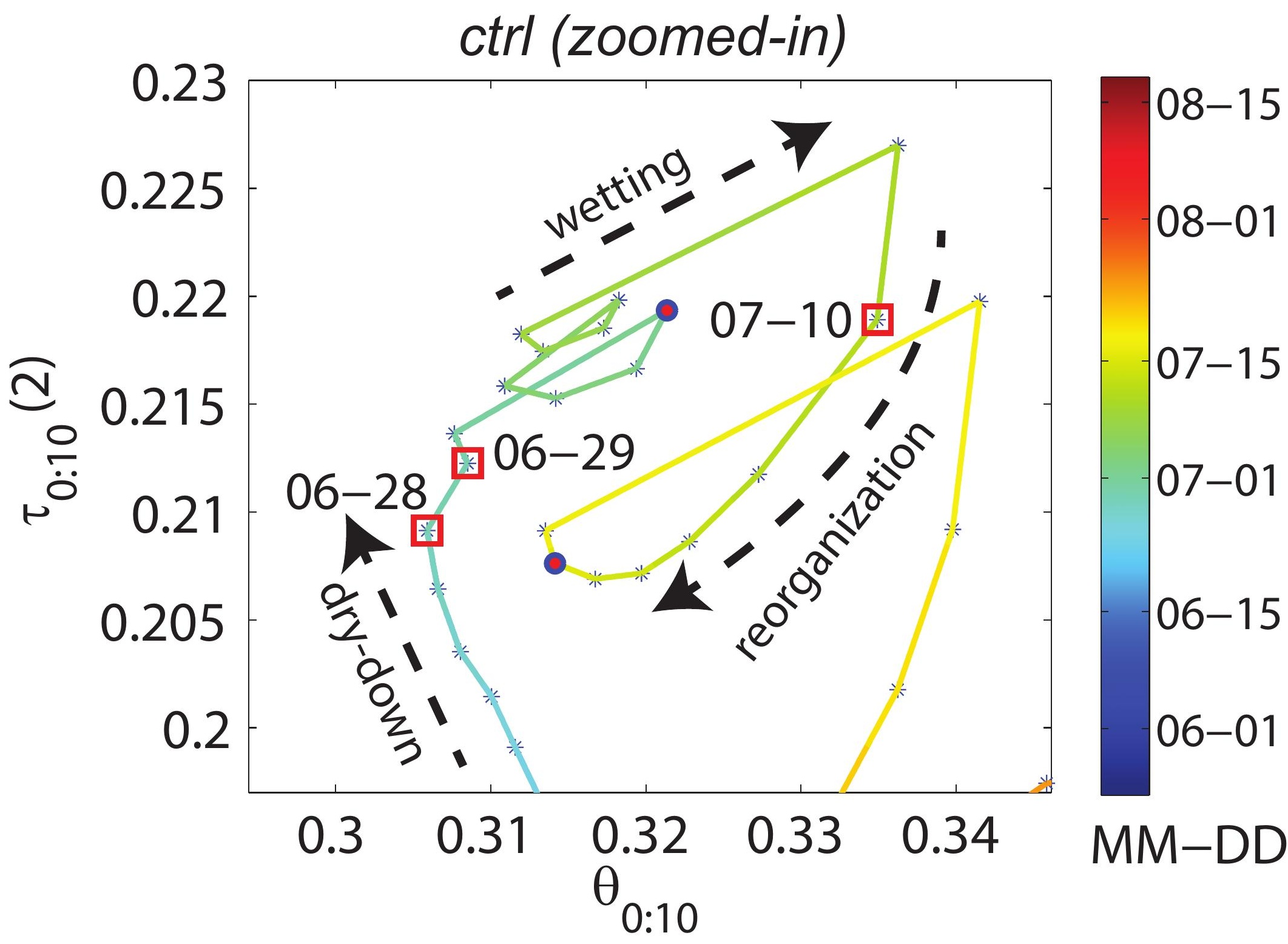


Figure07d

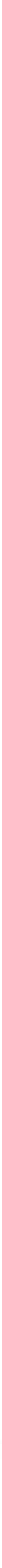

(
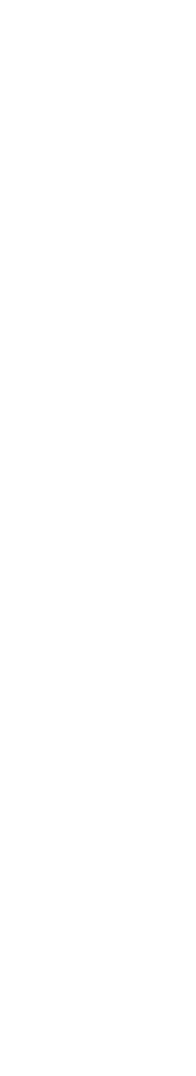

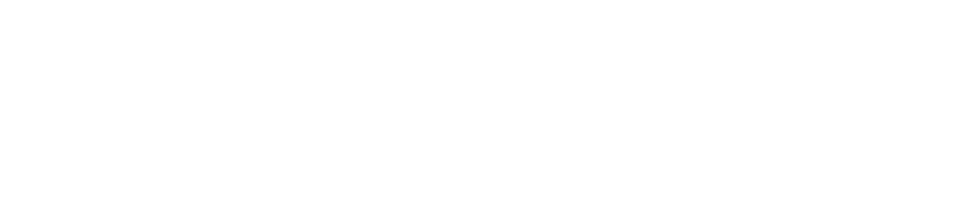



.

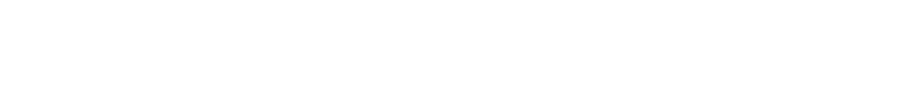

(

哟
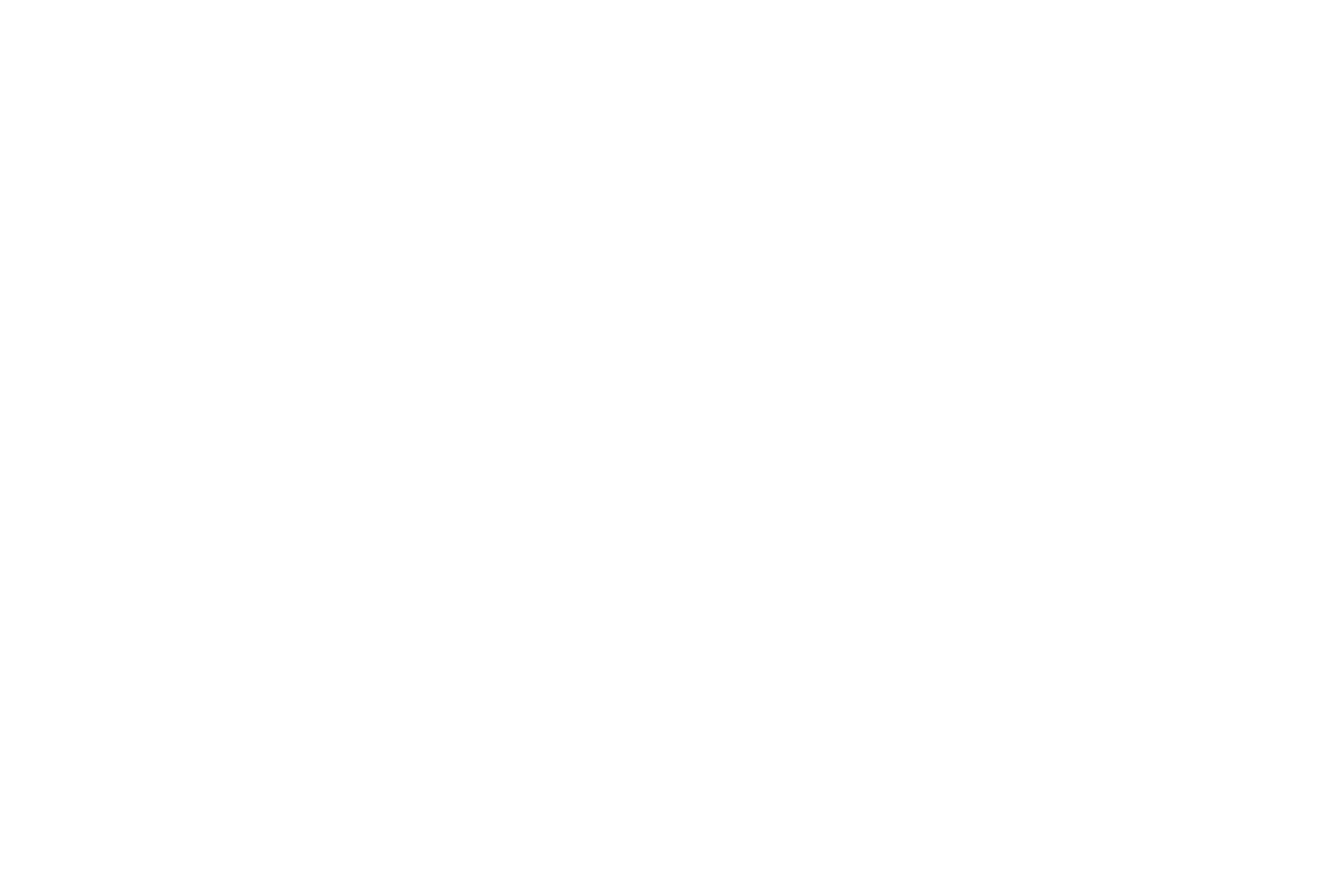
Click here to download Figure: Figure08a.eps

$\Delta \theta_{0: 10}(2005-06-28)$
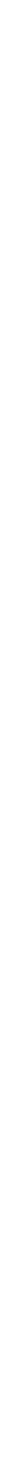
$\Delta \theta_{0: 10}(2005-06-29)$
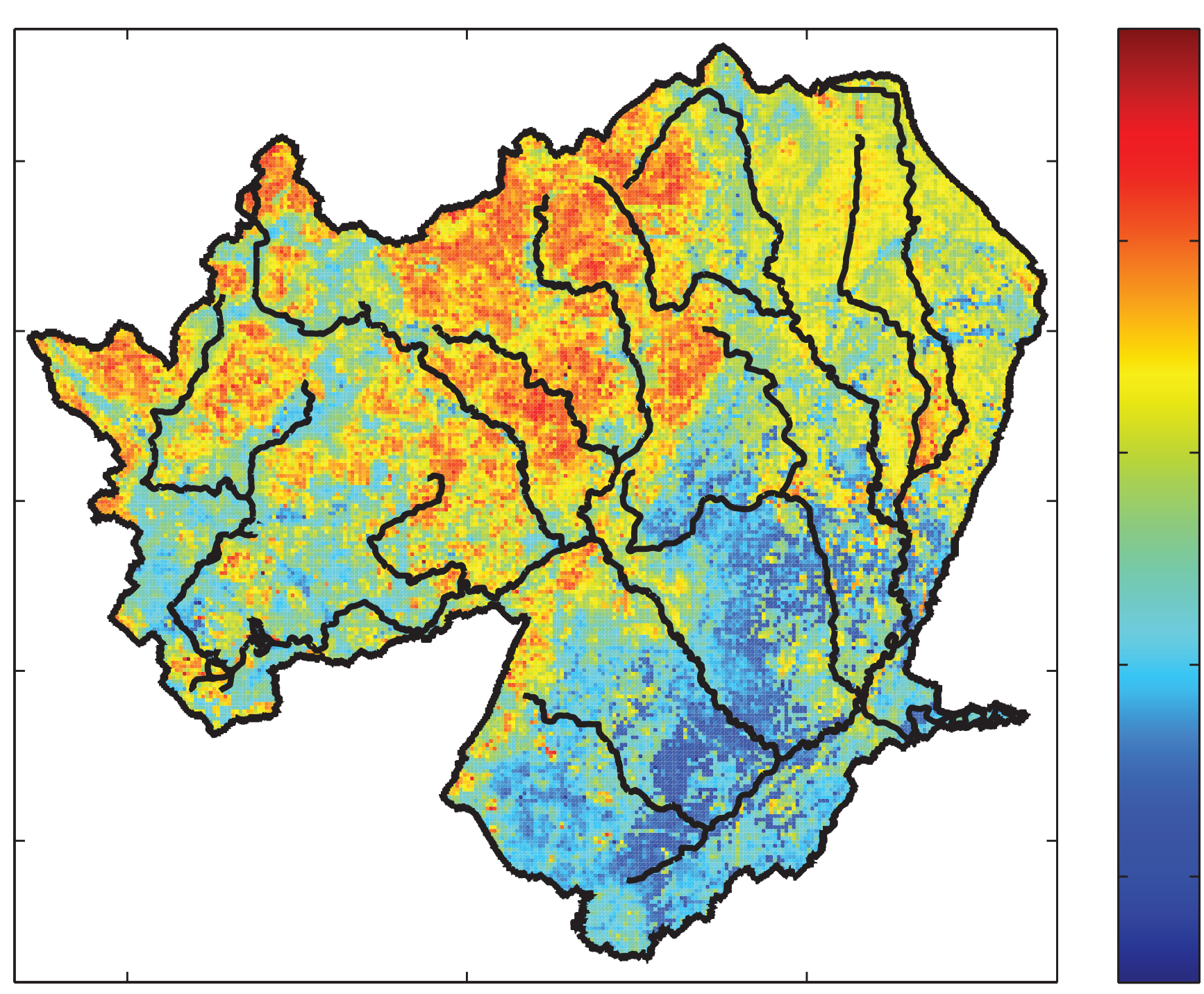

0.008

0.0058

0.0036

0.0014

$-0.0008$ 


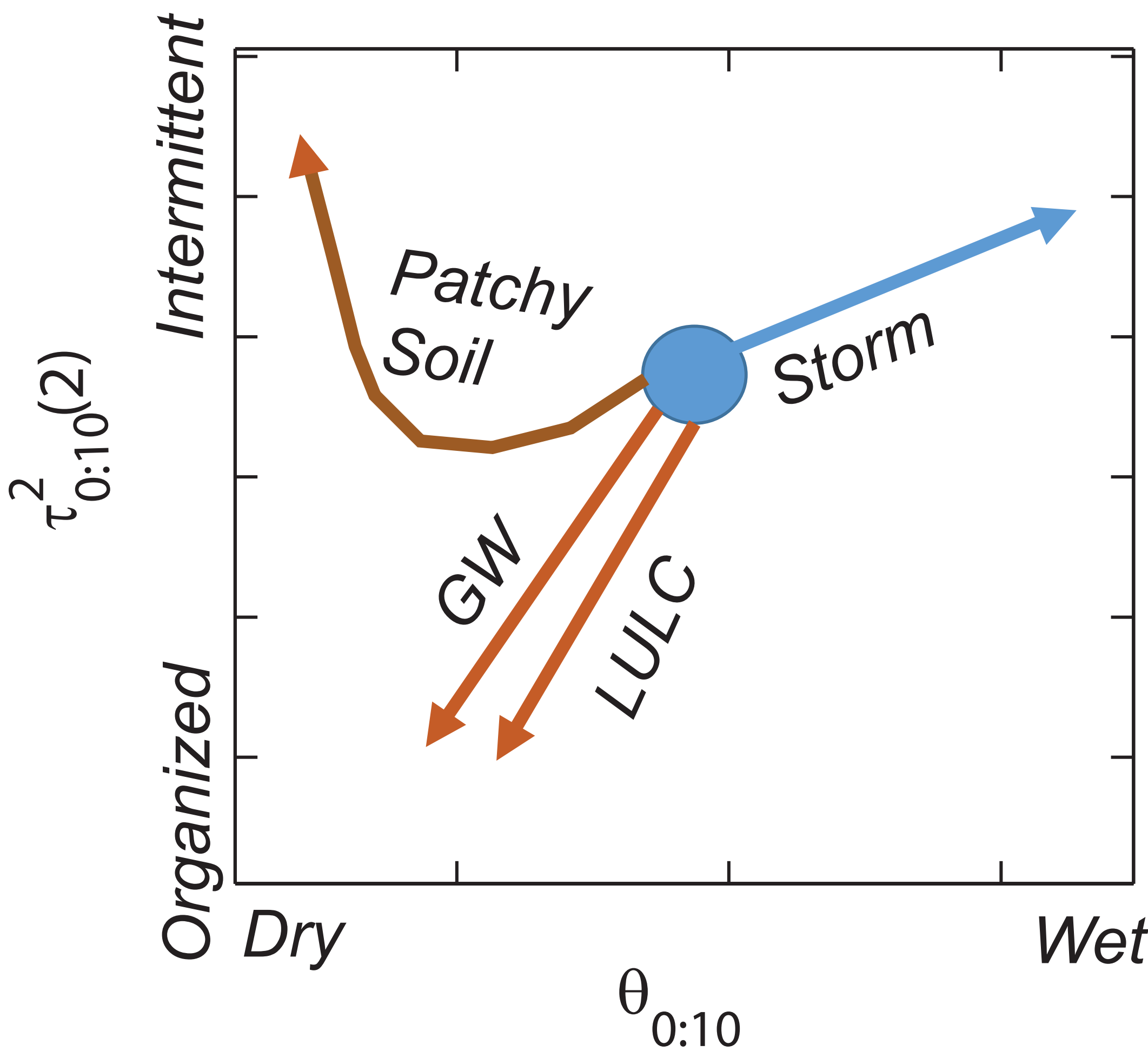


Color indicates $\overline{\theta_{0: 10}}$

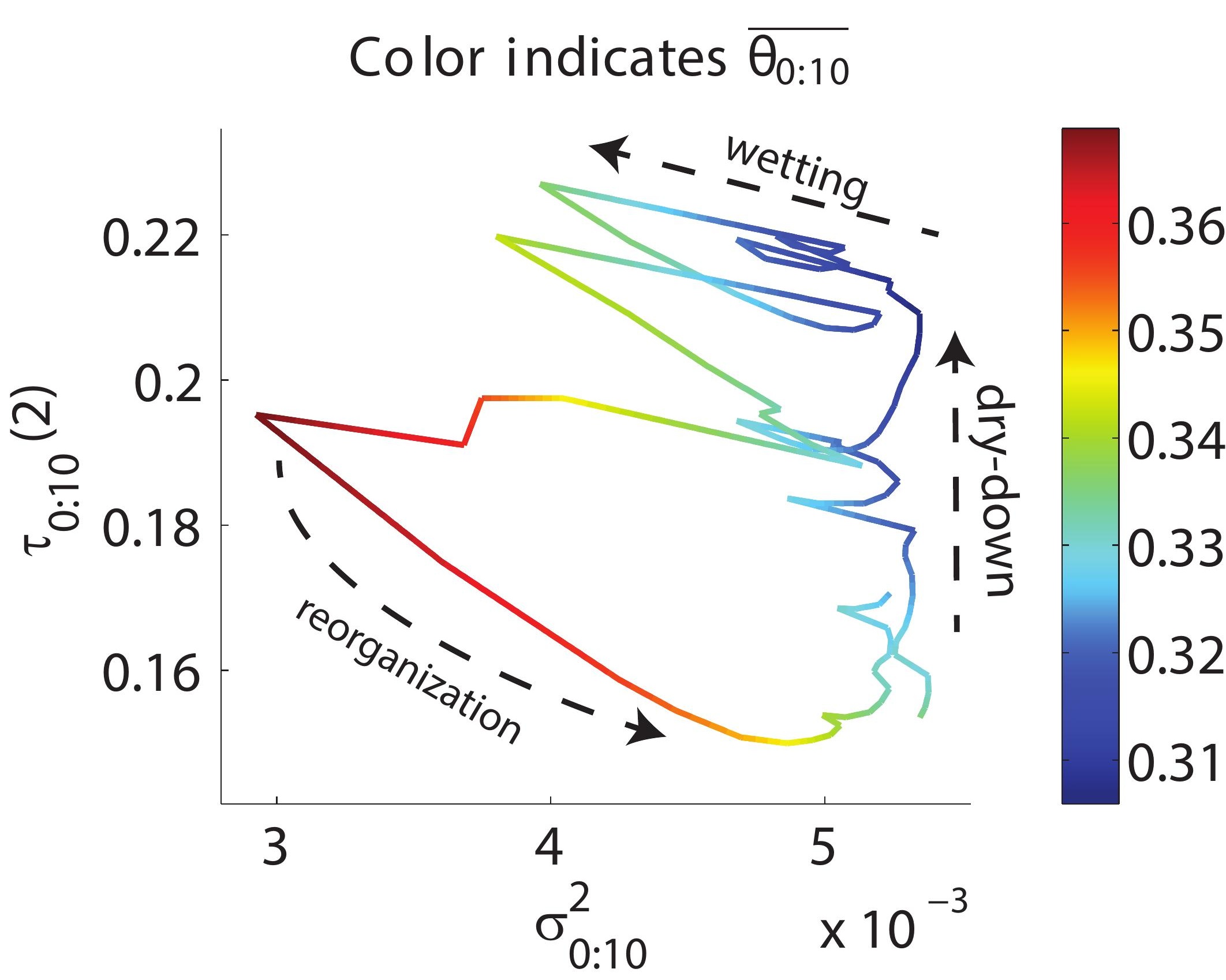

Figure11

0.31 


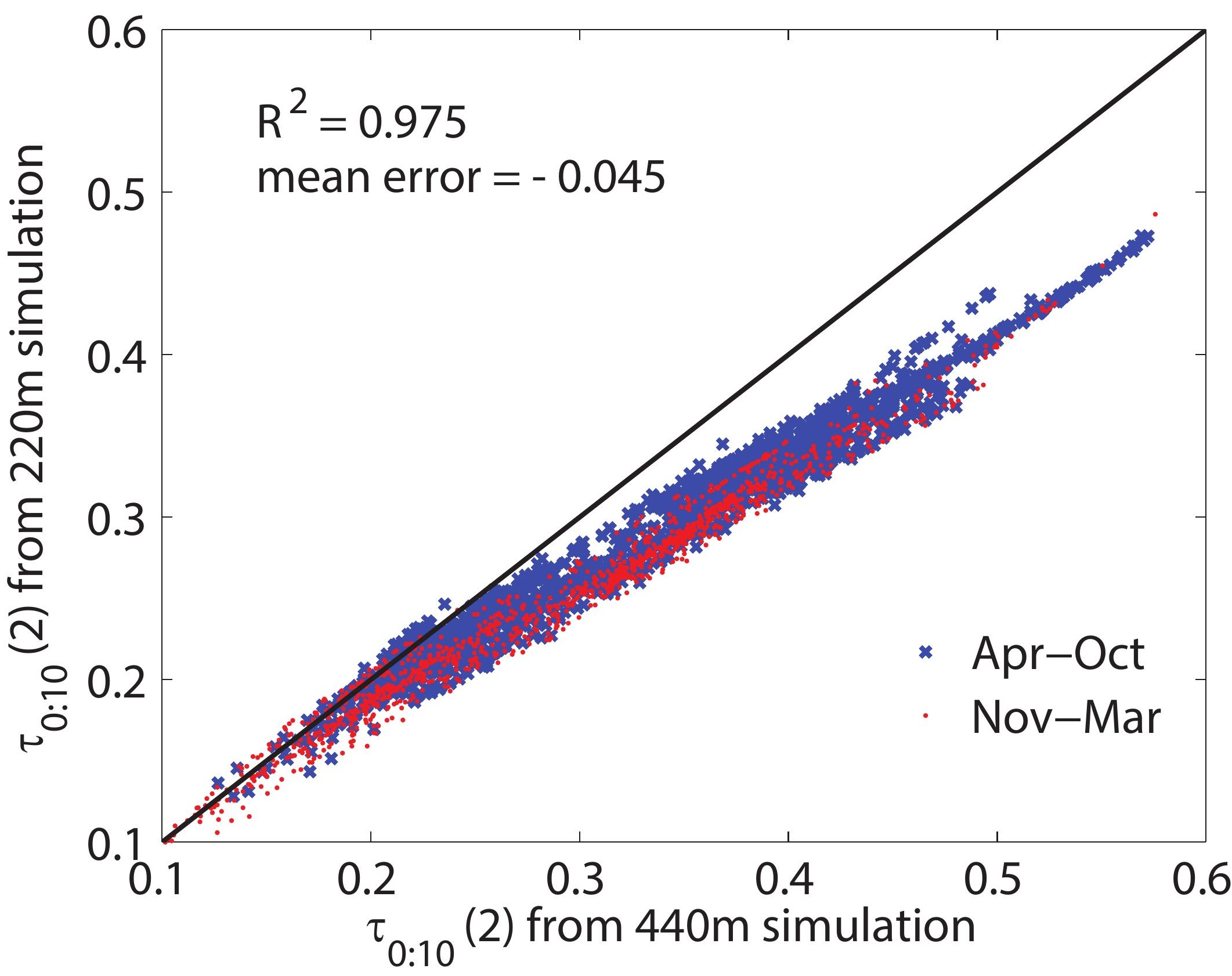




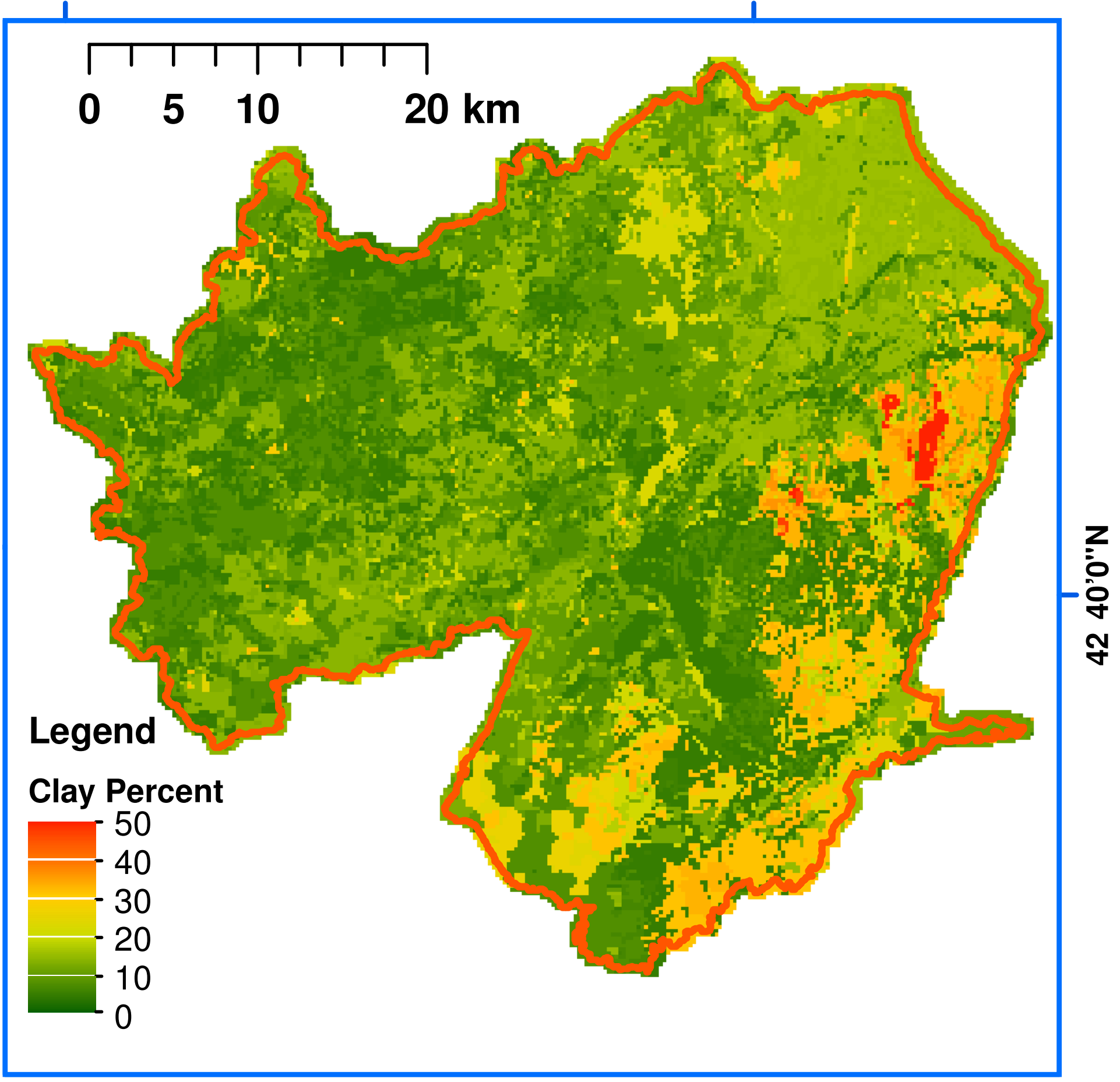


Click here to download Figure: FigureA2b.eps

\section{$\theta_{0: 10}$ (2005-Dec-01)}

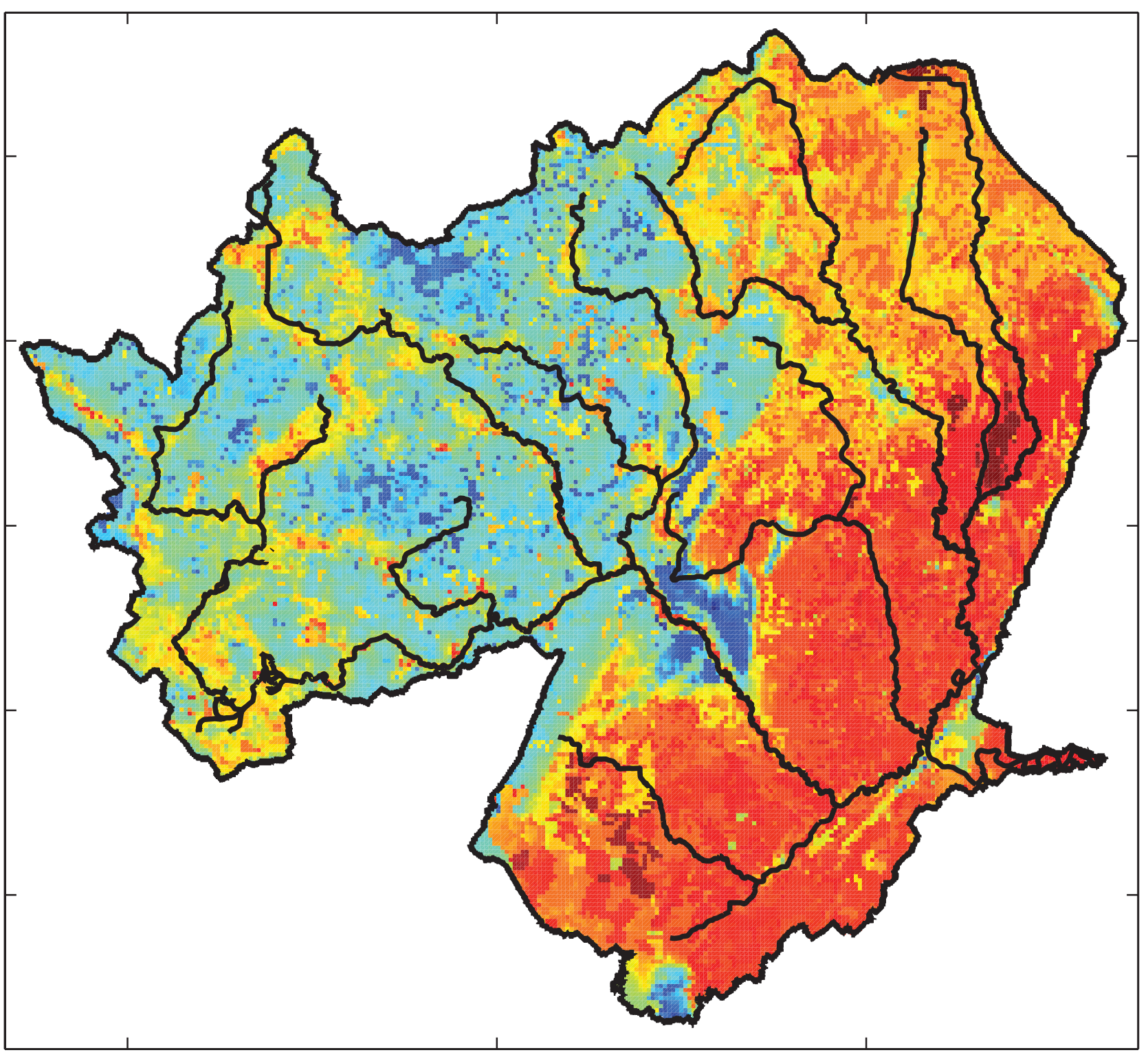

0.4959

0.4168

0.3378

0.2587

0.1797 
FigureA2c

2005-Jun-28

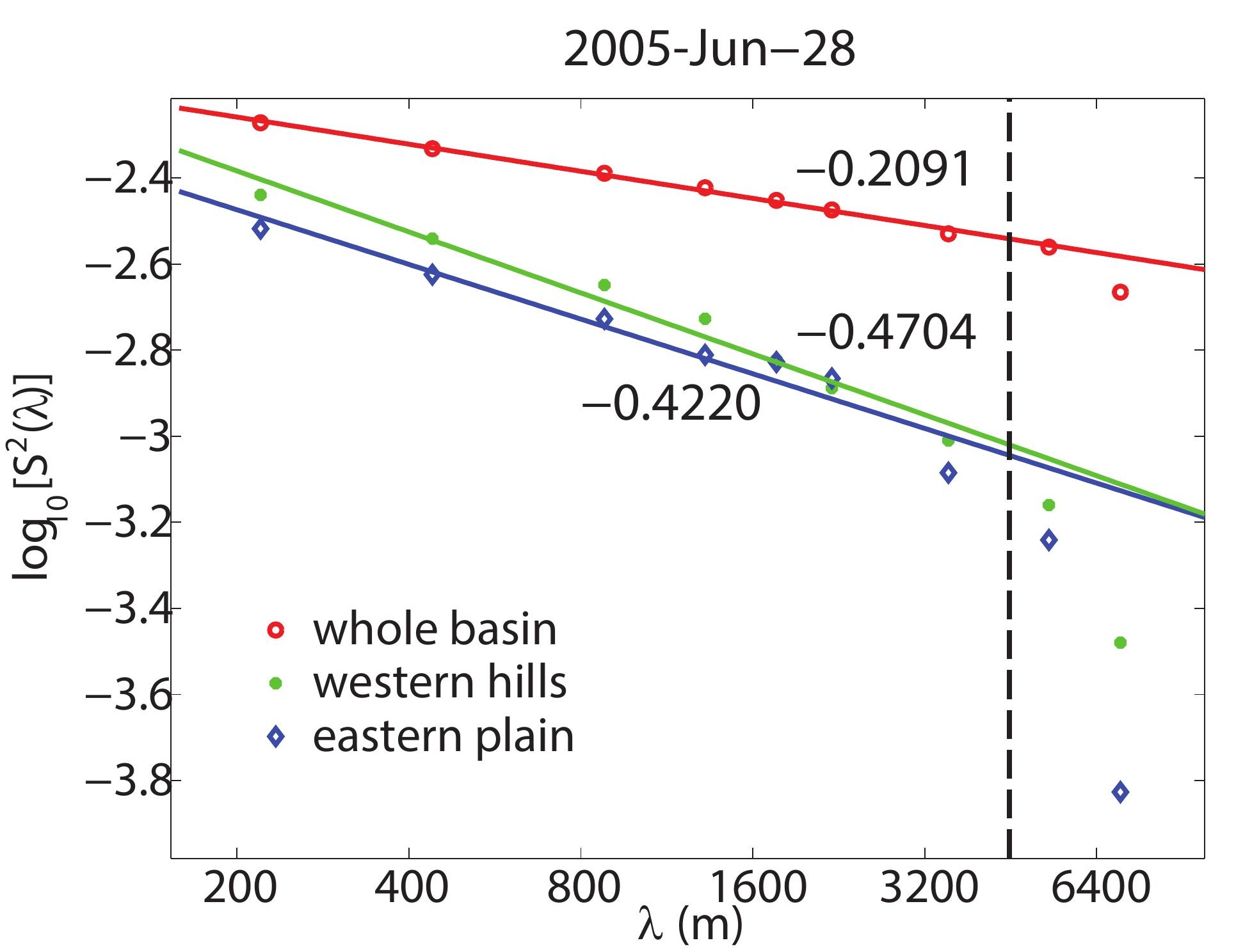


2005-Dec-01

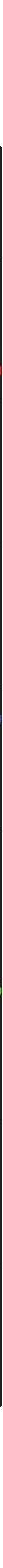

FigureA2d 
detect nonlinearity using robust regression

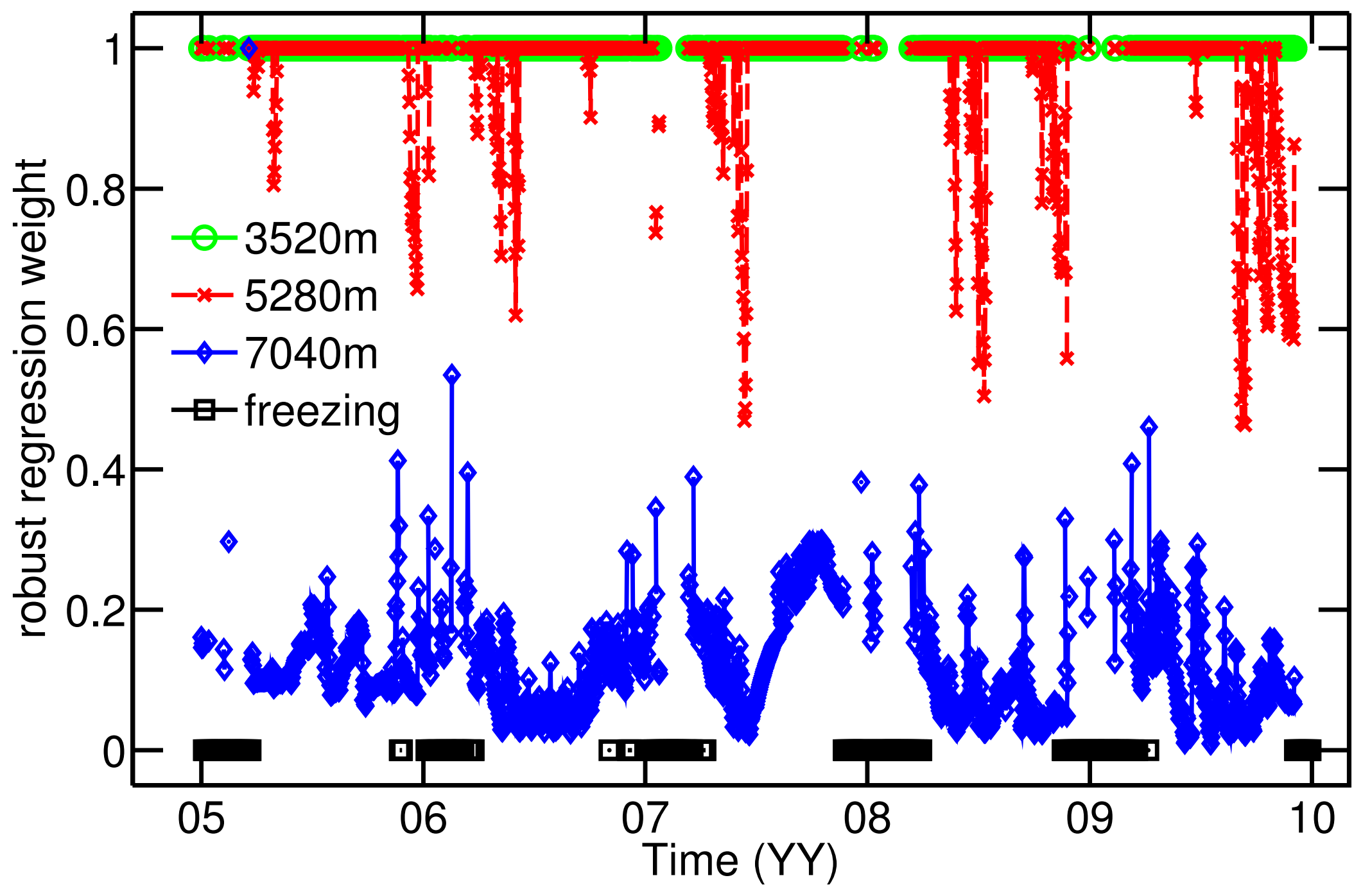




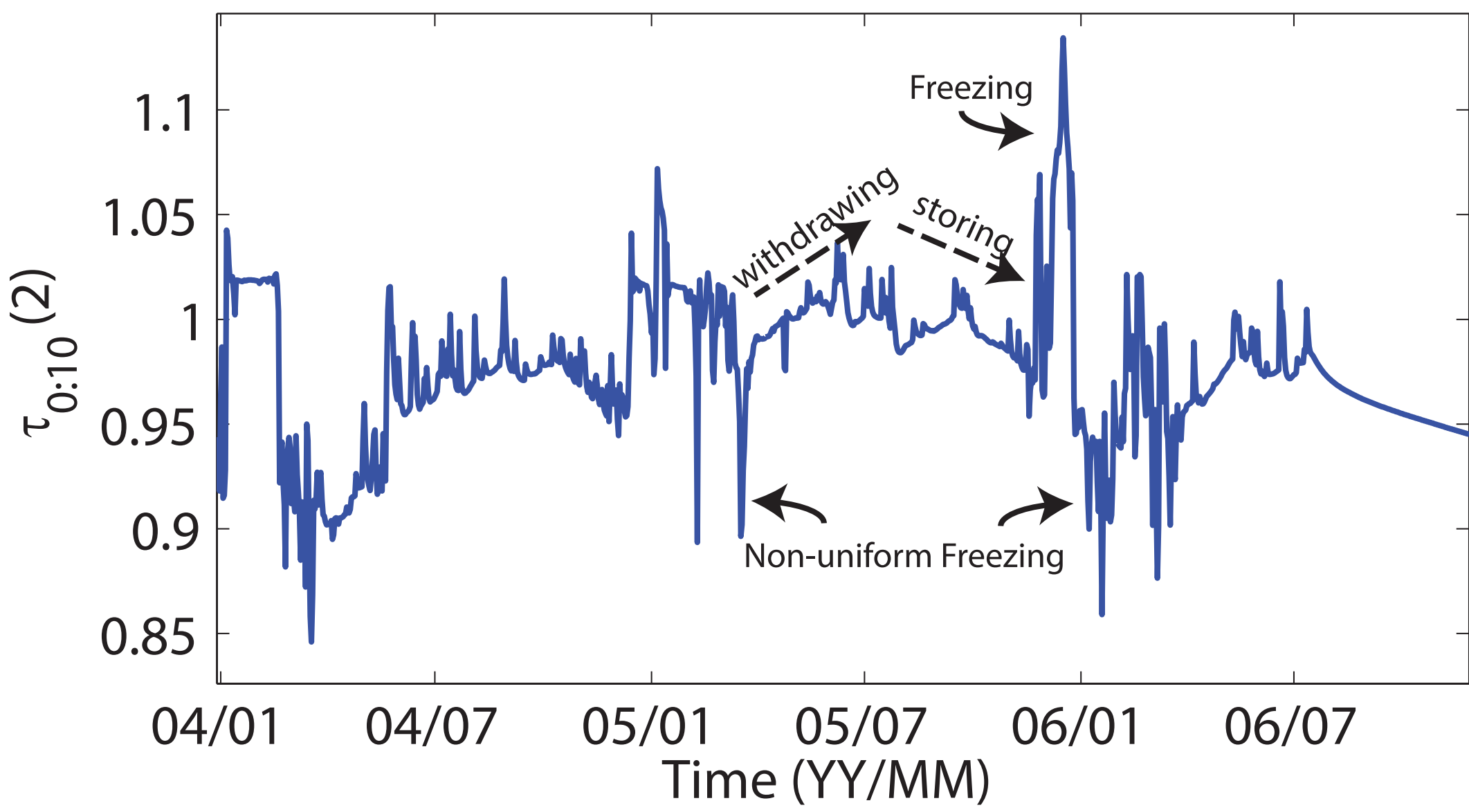






FigureA3b 